This item was submitted to Loughborough's Research Repository by the author.

Items in Figshare are protected by copyright, with all rights reserved, unless otherwise indicated.

\title{
Design of bifurcation junctions in artificial vascular vessels additively manufactured for skin tissue engineering
}

\section{PLEASE CITE THE PUBLISHED VERSION}

http://dx.doi.org/10.1016/j.jvlc.2014.12.005

\section{PUBLISHER}

(C) Elsevier Science Ltd

\section{VERSION}

AM (Accepted Manuscript)

\section{PUBLISHER STATEMENT}

This work is made available according to the conditions of the Creative Commons Attribution-NonCommercialNoDerivatives 4.0 International (CC BY-NC-ND 4.0) licence. Full details of this licence are available at: https://creativecommons.org/licenses/by-nc-nd/4.0/

\section{LICENCE}

CC BY-NC-ND 4.0

\section{REPOSITORY RECORD}

Han, Xiaoxiao, Richard J. Bibb, and Russell A. Harris. 2019. "Design of Bifurcation Junctions in Artificial Vascular Vessels Additively Manufactured for Skin Tissue Engineering”. figshare. https://hdl.handle.net/2134/18553. 


\title{
Design of Bifurcation Junctions in Artificial Vascular Vessels Additively Manufactured for Skin Tissue Engineering
}

\author{
Xiaoxiao Han*, Richard Bibb, Russell Harris
}

Additive Manufacturing Research Group, Loughborough University, Loughborough. Leicestershire, LE11 3TU, UK

*corresponding author, Email: x.han2@lboro.ac.uk, Tel: +44 (0)1509 227567

\begin{abstract}
Construction of an artificial vascular network ready for its additive manufacturing is an important task in tissue engineering. This paper presents a set of simple mathematical algorithms for computer-aided design of complex three dimensional vascular networks. Firstly various existing mathematical methods from the literature are reviewed and simplified for the convenience of applications in tissue engineering. This leads to a complete and step by step method for the construction of an artificial vascular network. Secondly a systematic parametric study is presented to illustrate how the various parameters in the vascular junction model affect the key factors that have to be controlled when designing the bifurcation junctions of a vascular network. These results are presented as a set of simple design rules and a design map which service as a convenient guide for tissue engineering researchers when constructing artificial vascular networks.
\end{abstract}

Keywords: additive manufacturing, 3D printing, vascular network design, bifurcation junction, junction smoothing, tissue engineering 


\section{Nomenclature}

A A set contains all vertices $\mathbf{Z}_{i}$ of a polygon hole

B Bi-normal unit vector in a Frenet frame

b $\quad \mathbf{b} \in \mathbf{M}$

$C_{\max }$ Maximum curvature of a bifurcation junction

$\bar{C}_{\max }$ non-dimensionalised $C_{\max }$

$D_{p} \quad$ Parent vessel diameter

$D_{d 1} \quad$ Daughter vessel diameter

$D_{d 2} \quad$ Daughter vessel diameter

$\bar{D}_{d 1} \quad$ non-dimensionlised $D_{d 1}$

$\bar{D}_{d 2} \quad$ non-dimensionlised $D_{d 2}$

$d_{p} \quad$ Definition shown in figure 4

$d_{d 1} \quad$ Definition shown in figure 4

$d_{d 2} \quad$ Definition shown in figure 4

$\bar{d}_{p} \quad$ Non-dimensionlised $d_{p}$

$\bar{d}_{d 1} \quad$ Non-dimensionlised $d_{d 1}$

$\bar{d}_{d 2} \quad$ Non-dimensionlised $d_{d 2}$

$F \quad$ The weight function in the hole triangulation method

$h_{1}, h_{2}, h_{3}$ Side length of a triangle

L Triangle perimeter

$\mathrm{l}_{\mathrm{c}}=\mathbf{S}_{d 1}\left(t, 90^{\circ}\right)$ 
$\mathrm{l}_{1}, \mathrm{l}_{2}, \mathrm{l}_{3}$ Boundaries of a curved bifurcation triangle

M Motion trajectories of a swept surface

N Normal unit vector in a Frenet frame

O Cubic Bezier matrix

$\mathbf{P}_{b} \quad$ Cubic Bezier points vector consisting of four control points

$\mathbf{P}_{0}, \mathbf{P}_{1}, \mathbf{P}_{2}, \mathbf{P}_{3}$ Four vectors of control points in a cubic Bezier curve

$\mathbf{Q}_{t} \quad$ Sweeping time vector in a cubic Bezier curve

$R_{1} \quad$ Radius of a cross sectional contour when $t=0$

$R_{2} \quad$ Radius of a cross sectional contour when $t=1$

$r \quad$ Radius of a cross sectional contour as a function of $t$

S A moving object used to construct a swept surface

$S \quad$ Triangle area

$\mathbf{S}^{\mathbf{b}} \quad$ A closed contour at position $\mathbf{b}$

$\mathbf{S}_{d 1} \quad$ A swept surface along trajectory $l_{2}$

Sweep A swept surface

$s \quad$ Arc length

T Tangent unit vector

$t \quad$ Sweeping time

$u_{i} \quad$ Distance of $\mathbf{P}_{i} \mathbf{P}_{i}{ }^{\prime}$ (Refer to figure (2a))

$u_{p}, u_{d 1}, u_{d 2}$ Control parameters

$\mathbf{v}_{\mathbf{p}}, \mathbf{v}_{d 1}, \mathbf{v}_{d 2}$ Unit vectors (Refer to figure (5))

$w_{i} \quad$ Distance of $\mathbf{P}_{i+1}$ and $\mathbf{P}_{i+1}{ }^{\prime}$ (Refer to figure (2a)) 
$\alpha=\frac{\min \left(\phi_{1}, \phi_{2}\right)}{\max \left(\phi_{1}, \phi_{2}\right)}$,

$\beta=\frac{\bar{D}_{d 1}}{\bar{D}_{d 2}}$

$\gamma \quad=\frac{\bar{d}_{d 1}}{\bar{d}_{d 2}}$.

$\omega \quad$ Contribution factor of $L$ in weight function $F$

$\varepsilon \quad$ Triangle quality coefficient

$\phi_{\text {total }}$ Bifurcation angle of a vascular branch

$\phi_{1}, \phi_{2}$ Bifurcation angle fractions 


\section{Introduction}

Many clinical therapies utilise autologous and allografts to repair skin defects resulting from genetic disorders, acute trauma, chronic wounds or surgical interventions. Tissue engineering (TE) of skin is an emerging technology that offers many potential advantages in repairing skin defects over conventional autologous grafts [1]. It overcomes the shortage of donor organs and reduces the added cost and complications of tissue harvesting. Tissue engineered skins can also be used as in vitro skin equivalent for pharmaceutical or cosmetics testing, eliminating the need for animal testing [2]. A major issue in tissue engineering is that the artificial skin may not develop adequate vascularisation for long-term survival [3]. Vascular vessels transport oxygen, nutrients and soluble factors to surrounding cells and tissues, without which cells or tissue would die [4]. Bio-artificial vascularised skin is thus of a great interest in tissue engineering. An artificial vascular system can be preembedded in a skin equivalent before it is implanted. The artificial vascular vessels not only provide nutrients and soluble factors to cells and tissues but also act as scaffolds for culturing vascular endothelial cells. Additive Manufacturing (AM) techniques, or 3D printing (3DP), produce physical objects directly from computeraided design (CAD) data in a layer-by-layer manner, and enable the manufacture of very small artificial vascular vessels required in skin tissue engineering [5]. "Artificial vascularised scaffolds for 3D-tissue regeneration (ArtiVasc 3D)" is a large project funded by the European Union's Seventh Framework Programme that aims to generate fully vascularised bioartificial skins. The work described in this paper is part of ArtiVasc 3D. The vascular structure, to be manufactured by a combination of micro-scale 3D printing (inkjet printing) or nano-scale stereolithography (multi-photon polymerisation), will be embedded in the adipose layer of a skin equivalent to supply 
nutrients and exchange waste [6]. In normal vasculature, around $98 \%$ of blood vessels bifurcate at each junction, while only $2 \%$ trifurcate $[7,8]$. Sharp apices at junctions of bifurcated vessels need to be avoided because they are considered as risk factors for local mechanical weakness [9]. Rounding (increasing the radius) the apex at each junction can be one of the solutions. However, larger recirculation areas of blood are found in bifurcation vessels with rounded apices compared with sharp junctions [9]. Thus a careful design of the bifurcation junctions is necessary.

Firstly this paper presents a simplification and integration of existing mathematical algorithms in the literature that can be conveniently used for modelling bifurcation junctions in tissue engineering. Unlike applications for vascular medical imaging, accurate replication of gross anatomy or patient specific anatomy is not required in tissue engineering. It is therefore possible to choose a set of simple algorithms. A parametric method is selected here that is free from generating bulges, selfintersections, unwanted inner surfaces or Boolean operations. Three-dimensional models of artificial vascular vessels generated using the method are ready to be translated into the triangular faceted STL (stereolithography) data file that drives the AM processes [10]. Secondly and more importantly this paper presents a systematic understanding in how the various parameters in the parametric model affect some of the key factors that have to be controlled when designing the bifurcation junctions. The study focuses on two key factors - the volume and maximum curvature of the junctions. The volume of the junction is important because an increased volume of the junction will cause larger local recirculation of blood [11, 12]. The maximum curvature is important because a high curvature generates high stress concentration and is considered as a mechanical risk. Furthermore the max curvature is the most important factor that influences the wall shear stress (WSS) $[9,12]$. The value range 
of maximum curvature at the apex is, however, very important and needs to be controlled carefully for certain applications. The paper presents a general design guide for constructing artificial vascular networks not only for skins but also for any other engineered tissues.

\section{Method for modelling vascular structures}

Modelling vascular structure has been an active field of research. Computer visualization of vascular structure plays a critical role in the diagnosis of vascular diseases, such as stenosis and aneurysm [12-14]. Computational fluids dynamics to study blood flow behaviour has to be based on accurate modelling of local vascular geometries $[11,12,14-16]$. Computer modelling of vascular bifurcation can be achieved in three ways: 1) skeleton based implicit surfaces [17-19], 2) blending objects obtained by canal surfaces $[20,21]$, and 3) sweeping disks or spheres along curve $[18,22,23]$. Unwanted inner self-intersecting surfaces and gaps may appear when sweeping full disks or spheres [18]. Implicit surface modelling can be used to address this problem as demonstrated by several researchers [17, 20, 25, 26]. However, Bulge problems are common when applying implicit surfaces to blend overlapping fields [17, 24-26]. Several strategies have been proposed to suppress them $[17,20,24,27]$, which increase the complexity of the model. The other two methods are usually used as a combined technique. Zakaria $\mathrm{H}$ et al [28] reported a three parameter model for constructing junctions of vascular network. Their method involves Boolean operations. A more general approach has been developed for blending canal surfaces by Krasauskas [21] using Pythagorean normal surfaces and by Bastl et al. [29] using a polyhedral medial structure. On the other hand, Cai et al, $[22,23]$ proposed a relatively simple method based on surface sweeping techniques. 
When blending canal surfaces, there is a major difference between applications in medical imaging and those in the design of artificial vascular network. In medical imaging, every details of the vascular geometry have to be captured accurately. In tissue engineering, on the other hand, one only needs to control some key factors when designing an artificial vascular network. It is therefore possible to select a simple method for the convenience of tissue engineering researchers. In this work the surface sweeping technique by Cai et al.[22, 23] is adopted. Cai et al. [22, 23] devised an algorithm to ensure cross-boundary tangential continuity when filling the holes left by the sweeping operation. This step complicates the modelling and is unnecessary for the purpose of designing an artificial vascular network. Here a simplified algorithm is presented with sufficient details for its full understanding.

\subsection{Sweeping a single tube}

Sweeping an object along a trajectory to obtain a solid surface or solid volume requires a moving object $\mathbf{S}$ and a motion trajectory $\mathbf{M}$ [30]. Equation (1) denotes a swept surface [30]:

$$
\operatorname{Sweep}(\mathbf{S} \text { on } \mathbf{M})=\bigcup_{\mathbf{b} \in \mathbf{M}} \mathbf{S}^{\mathbf{b}}
$$

where $\mathbf{S}$ represents the moving object, $\mathbf{M}$ is the motion trajectories and $\mathbf{S}^{\mathbf{b}}$ represents $\mathbf{S}$ positioned at position $\mathbf{b}$. The moving object $\mathbf{S}$ used to form vascular tubular vessels is a closed cross sectional contour. It can be either a fixed contour or a changing one from one shape to another along the trajectories. In the design of artificial vascular vessels, it is acceptable to assume $\mathbf{S}$ as a perfect circular contour. Linear interpolation is used to describe the varying radius of $\mathbf{S}$ along $\mathbf{M}$, i.e. we have 


$$
r(t)=R_{1}(1-t)+R_{2} t
$$

where $t \in[0,1]$ along $\mathbf{M}$ is the sweeping time, $R_{1}$ is the radius of the cross sectional contour when $t=0$ while $R_{2}$ is that when $t=1$.

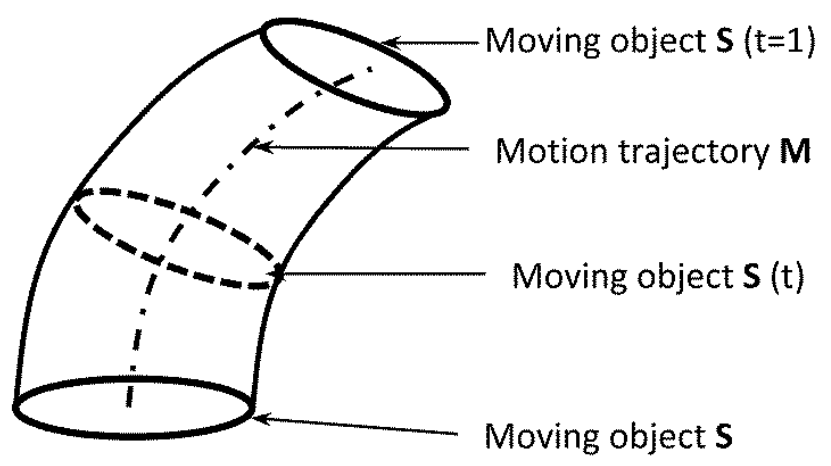

Figure 1. Schematic diagram of a swept surface with a moving object $\mathbf{S}$ and a motion trajectory $\mathbf{M}$; $t$ is the sweeping time.

Figure 1 illustrates schematically a sweeping surface created by moving $\mathbf{S}$ along $\mathbf{M}$. By using the Frenet-Serret frame or TNB frame, $\mathbf{S}(t)$ and $\mathbf{M}(t)$ can be expressed by equation (3):

$$
\begin{gathered}
\mathbf{M}(t)=\mathbf{P}(t) \\
\mathbf{S}(t, \theta)=\mathbf{P}(t)+\left(R_{1}(1-t)+R_{2} t\right)(\cos \theta \mathbf{N}(t)+\sin \theta \mathbf{B}(t))
\end{gathered}
$$

where $\mathbf{P}(t)$ is a curve in Euclidean space, representing the position vector of a particle as a function of time. In this paper, it is also used to represent the centre point position vector of a closed contour. $\mathbf{T}(t), \mathbf{N}(t)$ and $\mathbf{B}(t)$ are tangent, normal and bi-normal unit vectors of $\mathbf{P}(t)$, respectively [31]. $\theta$ is the angle that parameterizes a circle in the N, B frame. By substituting equation (3) into equation (1), an expression 
for a swept surface as a function of $t$ and $\theta$ can be obtained. By definition, $\mathbf{T}, \mathbf{N}$ and $\mathbf{B}$ are given by

$$
\mathbf{T}=\frac{d \mathbf{P}}{d s}, \mathbf{N}=\frac{\frac{d \mathbf{T}}{d s}}{\left\|\frac{d \mathbf{T}}{d s}\right\|}, \mathbf{B}=\mathbf{T} \times \mathbf{N}
$$

where $s$ represents the arc length along curve $\mathbf{P}$. A cubic Bezier curve is used to represent the trajectories $\mathbf{M}$ which is controlled by four points: $\mathbf{P}_{0}, \mathbf{P}_{1}, \mathbf{P}_{2}$, and $\mathbf{P}_{3}$. Thus curve $\mathbf{P}$ can be written as a function of $\mathbf{P}_{0}, \mathbf{P}_{1}, \mathbf{P}_{2}, \mathbf{P}_{3}$ and $t$ such that

$$
\mathbf{P}=\mathbf{Q}_{t}^{T} \mathbf{O} \mathbf{P}_{a},
$$

where

$$
\mathbf{Q}_{t}=\left[\begin{array}{c}
1 \\
t \\
t^{2} \\
t^{3}
\end{array}\right], \mathbf{O}=\left[\begin{array}{cccc}
1 & 0 & 0 & 0 \\
-3 & 3 & 0 & 0 \\
3 & -6 & 3 & 0 \\
-1 & 3 & -3 & 1
\end{array}\right], \mathbf{P}_{a}=\left[\begin{array}{c}
\mathbf{P}_{0} \\
\mathbf{P}_{1} \\
\mathbf{P}_{2} \\
\mathbf{P}_{3}
\end{array}\right]
$$

A constant time step $\Delta t$ does not give a constant arc-length for the Bezier curves of equation (5). Obtaining a uniform arc-length is important not only in a discretization process of a Bezier curve but also in the later triangulation section. Here Simpson integration and Newton methods are used to adjust $\Delta t$ for each time step to guarantee a uniform arc-length discretization. 


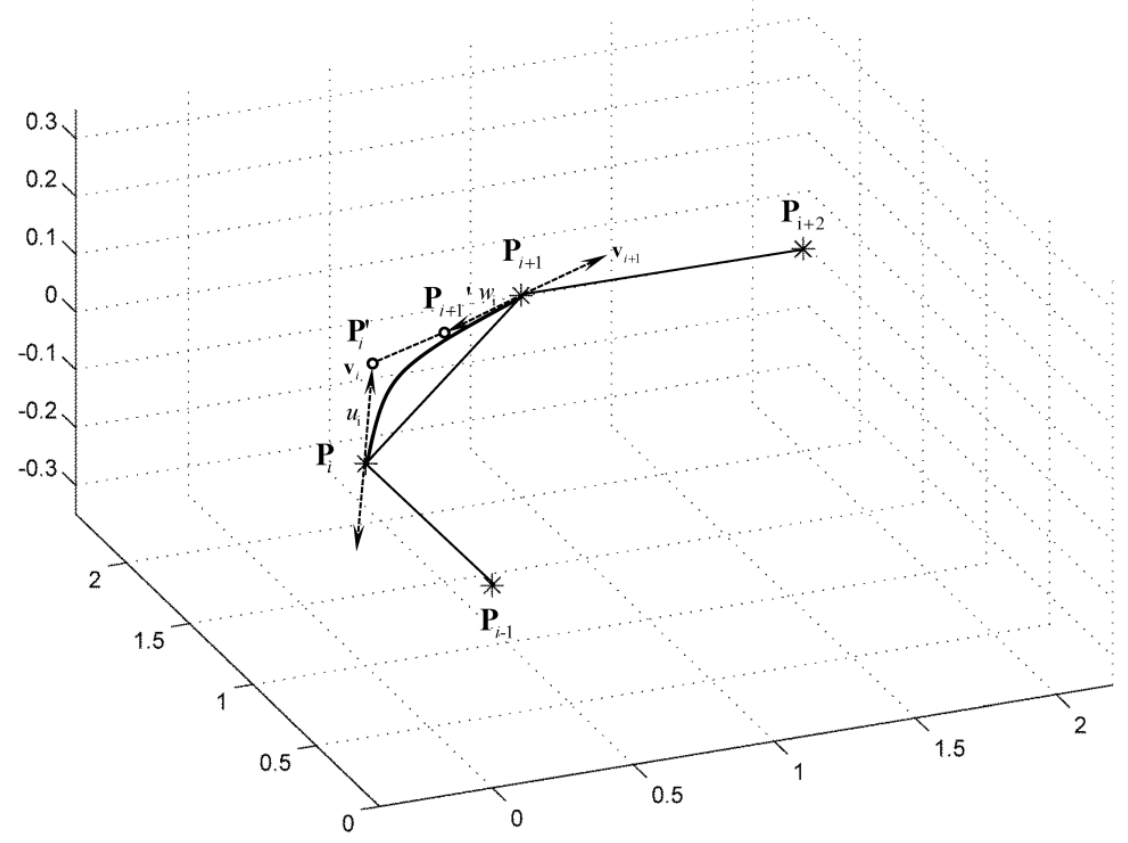

(a)

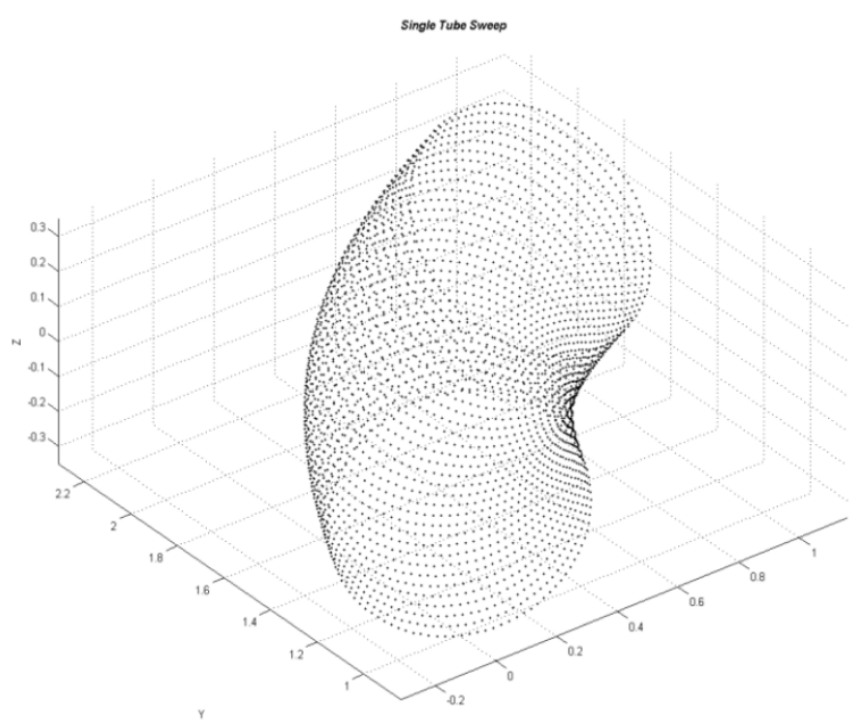

(b)

Figure 2. Variable definitions of a) cubic Bezier curve; and b) a cloud point of a swept surface. 
Figure 2 illustrates the steps of constructing a single tubular vessel from a known set of four points of $\mathbf{P}_{i-1}, \mathbf{P}_{i}, \mathbf{P}_{i+1}$ and $\mathbf{P}_{i+2}$. Here $\mathbf{P}_{i}$ and $\mathbf{P}_{i+1}$ are the start and end points respectively of a Bezier trajectory while $\mathbf{P}_{i-1}$ and $\mathbf{P}_{i+2}$ are used to derive unit vectors of $\mathbf{v}_{i}$ and $\mathbf{v}_{i+1}$ following the expressions given by equation (7):

$$
\mathbf{v}_{i}=\frac{\left(\mathbf{P}_{i+1}-\mathbf{P}_{i-1}\right)}{\left\|\mathbf{P}_{i+1}-\mathbf{P}_{i-1}\right\|}, \mathbf{v}_{i+1}=\frac{\left(\mathbf{P}_{i+2}-\mathbf{P}_{i}\right)}{\left\|\mathbf{P}_{i+2}-\mathbf{P}_{i}\right\|}
$$

The unit vectors $\mathbf{v}_{i}$ and $\mathbf{v}_{i+1}$ are used to guarantee $G^{1}$ continuity. To form a cubic Bezier curve, two intermediate points $\mathbf{P}_{i}$ ' and $\mathbf{P}_{i+1}{ }^{\prime}$ need to be generated. Their positions are determined by the unit vectors $\mathbf{v}_{i}$ and $\mathbf{v}_{i+1}$ and two controlled parameters $u_{\mathrm{i}}$ and $w_{\mathrm{i}}$. Here $u_{\mathrm{i}}$ and $w_{\mathrm{i}}$ are distances between $\mathbf{P}_{i}$ and $\mathbf{P}_{i}{ }^{\prime}$, and $\mathbf{P}_{i+1}$ and $\mathbf{P}_{i+1}{ }^{\prime}$, respectively. All the notations are shown in figure 2(a). $\mathbf{P}_{a}$, representing $a$ control set with four vertices from equation (6), is therefore formed. A single tube swept surface represented by cloud points, obtained using equations (3) to (6), is shown in figure 2(b).

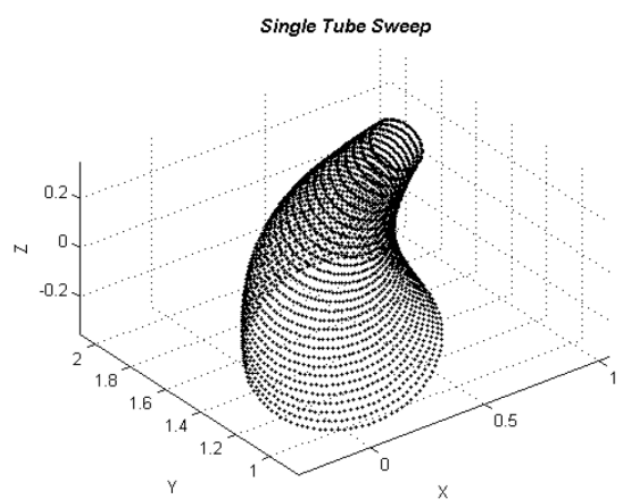


Figure 3. A swept surface by sweeping a changing circular cross sectional contour along a cubic Bezier developed in figure 2(b) using a linear interpolation method provided in equation (2).

Figure 3 shows a swept surface using varied radius: radius of the moving circle reduces from $0.35 \mathrm{~mm}$ to $0.1 \mathrm{~mm}$ following the linear interpolation of equation (2).

\subsection{Construction of the branch junctions}

Designing a bifurcation junction involves the following steps:

\subsubsection{Finding a bifurcation triangle for the junction}

A bifurcation junction contains a parent vessel, two daughter vessels and a bifurcation point. Assuming the parent vessel diameter is known, contour diameters of two daughter vessels can be obtained from Murray's law [32]. Central spine lines, diameters of the parent vessel and its two daughter vessels as well as the bifurcation point are shown in figure 4.

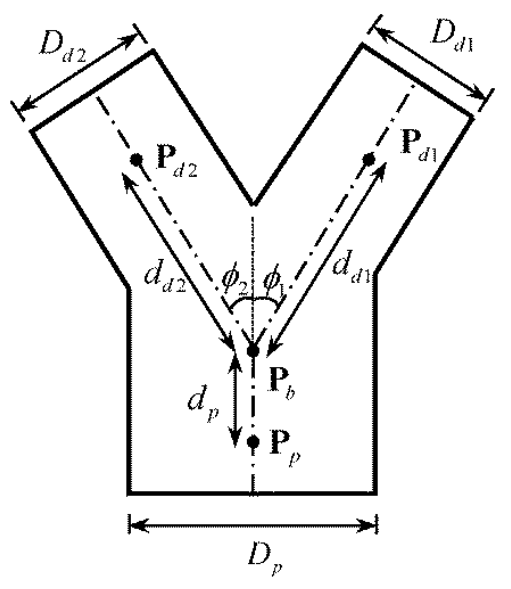

Figure 4. A schematic diagram for a bifurcation junction and variable definitions.

In the figure, $D_{P}, D_{d 1}$ and $D_{d 2}$ are diameters of the parent vessel and two daughter vessels respectively. $\mathbf{P}_{b}$ is the bifurcation point from which a parent vessel sprouts 
into its two daughter vessels. $\phi_{1}$ and $\phi_{2}$ are the bifurcation angles for the two daughter vessels. The three vertices $\left(\mathbf{P}_{p}, \mathbf{P}_{d 1}\right.$ and $\left.\mathbf{P}_{d 2}\right)$ of the junction triangles can be defined by their distances to $\mathbf{P}_{b}$, i.e. we have $d_{p}=\mathbf{P}_{p} \mathbf{P}_{b}, d_{d 1}=\mathbf{P}_{d 1} \mathbf{P}_{b}$ and $d_{d 2}=\mathbf{P}_{d 2} \mathbf{P}_{b}$. The planar triangle $\mathbf{P}_{p} \mathbf{P}_{d 1} \mathbf{P}_{d 2}$ is referred to as a straight bifurcation triangle. Murray's law [32] dictates that

$$
D_{P}{ }^{3}=D_{d 1}{ }^{3}+D_{d 2}{ }^{3}
$$

\subsubsection{Establishing cubic Bezier curves for the bifurcation triangle}

Three cubic Bezier curves are used to reconstruct the straight bifurcation triangle into its 3D curved counterpart. These three curves will be used as the trajectories of the three swept surfaces. Figure 5 shows the bifurcation triangle $\mathbf{P}_{p} \mathbf{P}_{d 1} \mathbf{P}_{d 2}$ and its corresponding unit vectors $\mathbf{v}_{p}, \mathbf{v}_{d 1}$ and $\mathbf{v}_{d 2}$.

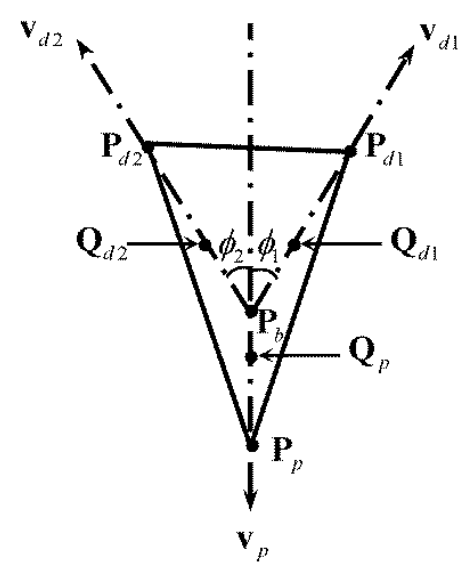

Bifurcation triangle

Figure 5. A bifurcation triangle $P_{p} P_{d 1} P_{d 2}$, corresponding unit vectors: $v_{p}, v_{d 1}$ and $v_{d 2}$, three control points: $Q_{p}, Q_{d 1}$ and $Q_{d 2}$, and bifurcation angles: $\phi_{1}$ and $\phi_{2}$. 
Recall that constructing a cubic Bezier curve requires four points: $\mathbf{P}_{i}, \mathbf{P}_{i}{ }^{\prime}, \mathbf{P}_{i+1}{ }^{\prime}, \mathbf{P}_{i+1}$, in which $\mathbf{P}_{i}$ and $\mathbf{P}_{i+1}$ are start and end points, $\mathbf{P}_{i}^{\prime}$ and $\mathbf{P}_{i+1}{ }^{\prime}$ are control points respectively (figure 2(a)). $\mathbf{P}_{i}^{\prime}$ and $\mathbf{P}_{i+1}{ }^{\prime}$ are dependent on controlled parameters $u_{\mathrm{i}}$ and $w_{\mathrm{i}}$, and direction vectors $\mathbf{v}_{i}$ and $\mathbf{v}_{i+1}$. In a straight bifurcation triangle, three cubic Bezier curves can be generated: $\mathrm{l}_{1}, \mathrm{l}_{2}$ and $\mathrm{l}_{3}$, based on control points $\left[\begin{array}{c}\mathbf{P}_{p} \\ \mathbf{Q}_{p} \\ \mathbf{Q}_{d 1} \\ \mathbf{P}_{d 1}\end{array}\right],\left[\begin{array}{c}\mathbf{P}_{d 1} \\ \mathbf{Q}_{d 1} \\ \mathbf{Q}_{d 2} \\ \mathbf{P}_{d 2}\end{array}\right]$ and $\left[\begin{array}{c}\mathbf{P}_{d 2} \\ \mathbf{Q}_{d 2} \\ \mathbf{Q}_{p} \\ \mathbf{P}_{p}\end{array}\right]$. Three control points $\mathbf{Q}_{p}, \mathbf{Q}_{d 1}, \mathbf{Q}_{d 2}$ displayed in figure 5 are calculated based on unit vectors $\mathbf{v}_{p}, \mathbf{v}_{d 1}, \mathbf{v}_{d 2}$ and three control parameters $u_{p}, u_{d 1}, u_{d 2}$, each of which varies from 0 to 1 . Therefore we have:

$$
\mathbf{Q}_{p} \mathbf{P}_{p}=u_{p} \times \mathbf{P}_{p} \mathbf{P}_{b}, \mathbf{Q}_{d 1} \mathbf{P}_{d 1}=u_{d 1} \times \mathbf{P}_{d 1} \mathbf{P}_{b}, \mathbf{Q}_{d 2} \mathbf{P}_{d 2}=u_{d 2} \times \mathbf{P}_{d 2} \mathbf{P}_{b}
$$

Despite the straight bifurcation triangle is strictly planar, the bifurcation curved triangle may not be planar because unit vectors $\mathbf{v}_{p}, \mathbf{v}_{d 1}, \mathbf{v}_{d 2}$ can be non-planar.

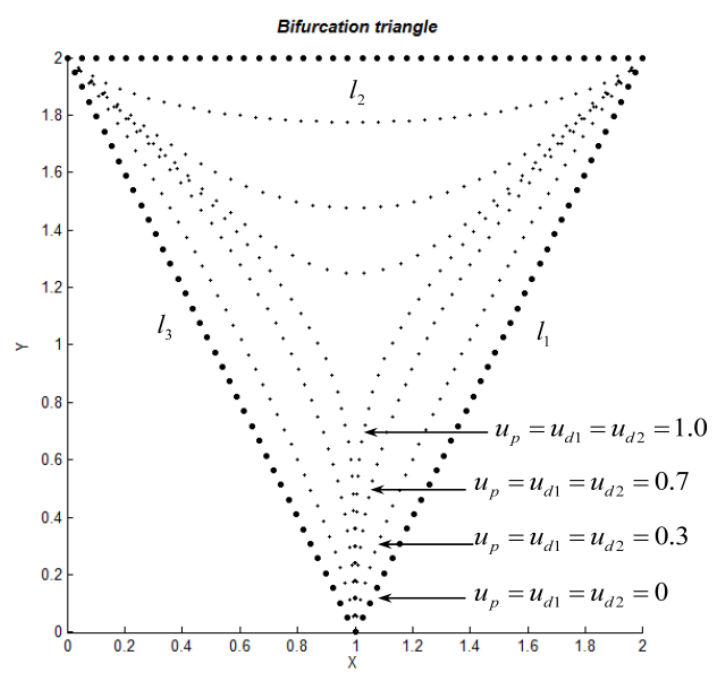


Figure 6. Curved bifurcation triangles $\left(l_{1}, l_{2}, l_{3}\right)$ representing by cubic Bezier curves with different sets of control parameters: from outer triangle to inner triangles, values of control parameters, in turn, are $u_{p}=u_{d 1}=u_{d 2}=0, u_{p}=u_{d 1}=u_{d 2}=0.3$,

$$
u_{p}=u_{d 1}=u_{d 2}=0.7, \text { and } u_{p}=u_{d 1}=u_{d 2}=1.0 .
$$

Figure 6 shows a set of bifurcation triangles with initial vertices at $\mathbf{P}_{p}(1,0,0)$, $\mathbf{P}_{d 1}(2,2,0), \mathbf{P}_{d 2}(0,2,0)$ and a bifurcation point at $\mathbf{P}_{b}(1,1,0)$. The four sets of cubic Bezier curves are obtained using different control parameters. Setting $u_{p}=u_{d 1}=u_{d 2}=1$ yields a curved triangle with minimal area and maximum curvature of each edge. The maximum curvature always occurs on $1_{c}$ because of the limited range of the bifurcation angle in vascular bifurcation networks [9].

\subsubsection{Constructing bifurcation junctions}

Cai et al, [22] introduced a semi-tubular sweeping method for constructing junctions where three canal surfaces meet together. In this method, only a half disk of each cross sectional contour is swept along three trajectories to form the outer surface of a junction, thus avoiding any overlapping, self-intersecting or unwanted inner surfaces. Figure 7 shows a bifurcation junction obtained using this method. Cai et al. [22] further proposed an algorithm to fill the two polygon holes left after the sweeping step. 


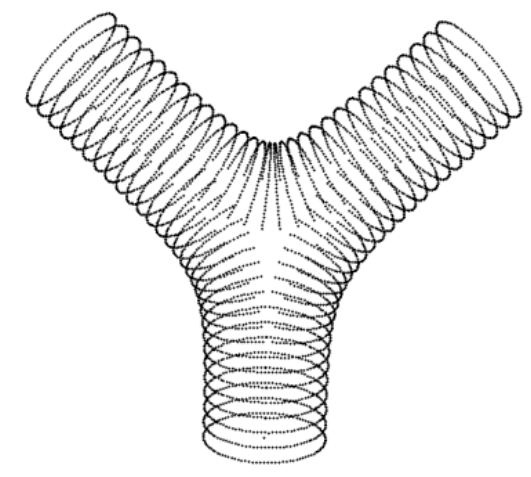

Figure 7. The swept semi-tubular bifurcation junction.

In particular their method ensures the cross-boundary tangential continuity between the hole filling patches and outer surface of the tubes. This step is rather complicated for tissue engineering researchers. The issue of cross-boundary tangential continuity is not significant in the practical applications for artificial vascular network. This will be demonstrated using examples in the later part of this paper. We therefore propose to use a simple triangulation method $[33,34]$ for the hole filling step. This method also has the advantage of being consistent with the triangulation data structure used by the STL file that drives additive manufacturing.

Triangulation is a standard meshing technique that uses a set of small triangles to fill up a surface area with predefined boundaries. Very long and narrow triangles are problematic in subsequent operations [33]. Several algorithms are available that optimizes some measure of quality of the mesh $[33,34]$. We chose a nondimensional parameter $\varepsilon$ to measure the quality of the triangles which is defined as

$$
\varepsilon=\frac{4 S \sqrt{3}}{h_{1}^{2}+h_{2}^{2}+h_{3}^{2}},
$$

in which $S$ is the triangle area and $h_{1}, h_{2}$ and $h_{3}$ are the side lengths of a triangle. For an equilateral triangle (the best) $\varepsilon=1$ and for other triangles $\varepsilon$ is in the range of 
$(0,1]$. Long and narrow (bad) triangles have smaller values of $\varepsilon$. To construct a mesh with high values of $\varepsilon$, a weight function $F$ is defined on each triangle such that

$$
F=S+\omega L
$$

in which $L$ is the perimeter of a triangle and $\omega$ is empirical weighting factor. A small value of $\omega$ less than 0.02 is sufficient to ensure good mesh quality. The triangulation problem thus becomes to find a triangulation mesh that minimizes the total sum of F over its triangles [33].

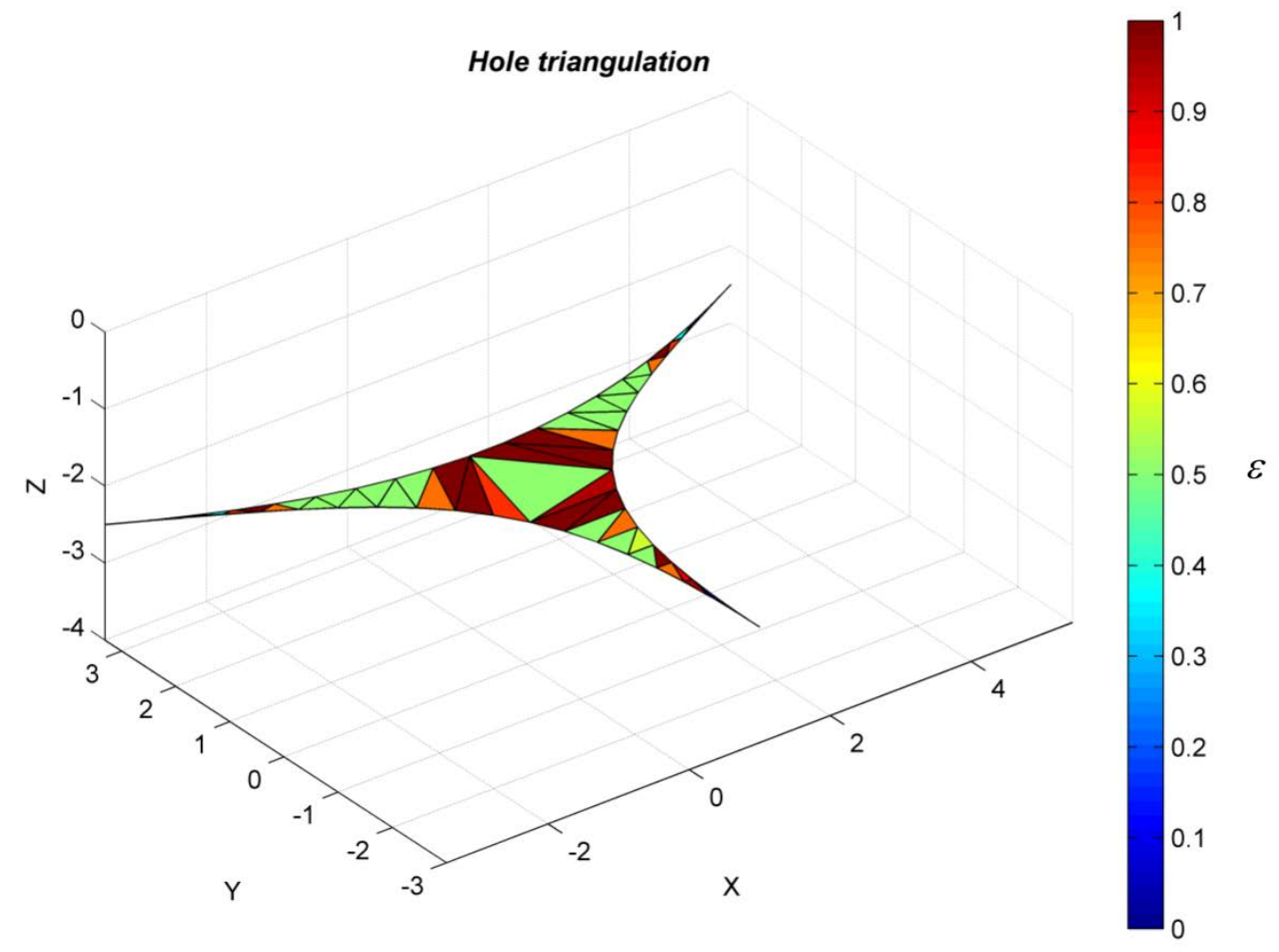

Figure 8. A polygon hole filling of a planar hole $\omega=0.01, \varepsilon=0.5494$. 
Figure 8 shows an example for filling a planar hole using $\omega=0.01$. The average values of $\varepsilon$ is 0.5494 with most of the triangle having $\varepsilon>0.5$.

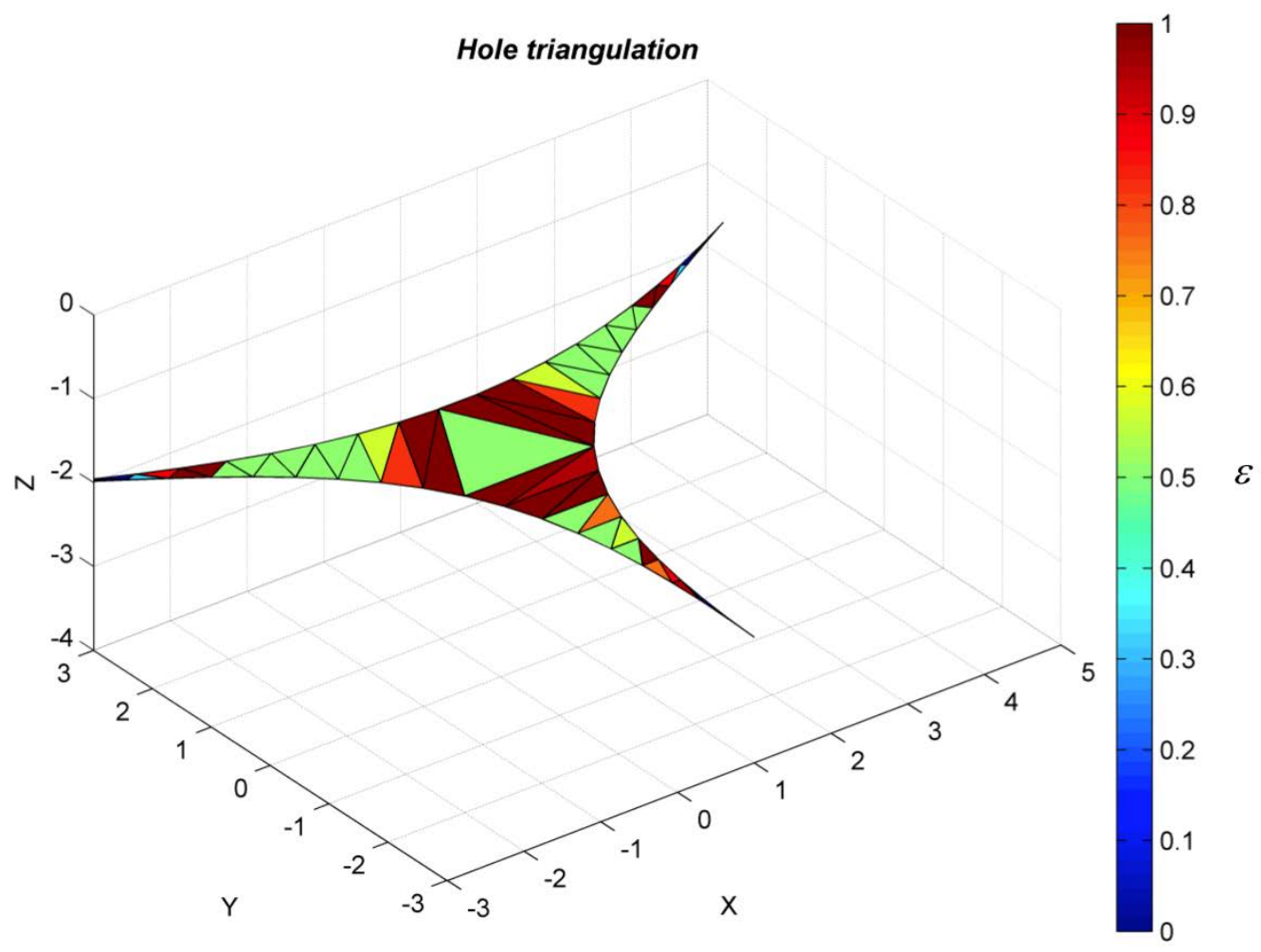

Figure 9. A polygon hole filling of a non-planar hole $\omega=0.01, \varepsilon=0.5789$.

Figure 9 shows an example of filling a non-planar hole with $\omega=0.01$. The average values of $\varepsilon$ is 0.5789 .

\subsubsection{Wall thickness generation}

Generating the wall thickness can be easily achieved by generating two surface models with different parent diameters: $D_{P}$ and $D_{P}+$ (wall_thickness). Daughter vessels' wall thicknesses can be obtained by applying two diameters as well. There are two ways to calculate the outer diameters of daughter vessels which are expressed by: 


$$
D_{d}^{3}=\frac{\left(D_{P}+\text { wall_thickness }\right)^{3}}{2}
$$

and

$$
D_{d}{ }^{3}=\frac{\left(D_{P}\right)^{3}}{2}+(\text { wall_thickness })^{3} .
$$

Equation (12) gives a non-uniform wall thickness using which the whole networks have different wall thicknesses at each branching level while equation (13) generates uniform wall thickness for the whole networks. In practice, variational wall thickness (equation (12)) always applies.

\subsection{Translating the models to STL files}

The STL (StereoLithography) file format is the de-facto standard for additive manufacturing [10]. It is a surface representation of solid models and generated by using a large number of triangles. STL is a linear approximation of a smooth surface, therefore when translating from a given smooth curved surface to a STL file format, approximation always occurs. The mathematical methods described in the previous sections generate two types of data: smooth surfaces of the three semi-swept tubes and the polygon holes represented by triangles. The latter can be translated into STL file format simply by reordering the three vertices using the right-hand rule and calculating the unit facet normal for each triangle. In order to translate the former to STL file format, one can stitch discretization points on matching borders to form ordered triangles which is fully described in ref [34]. Figure 10 provides an example of a STL mesh translated from the surface model. 


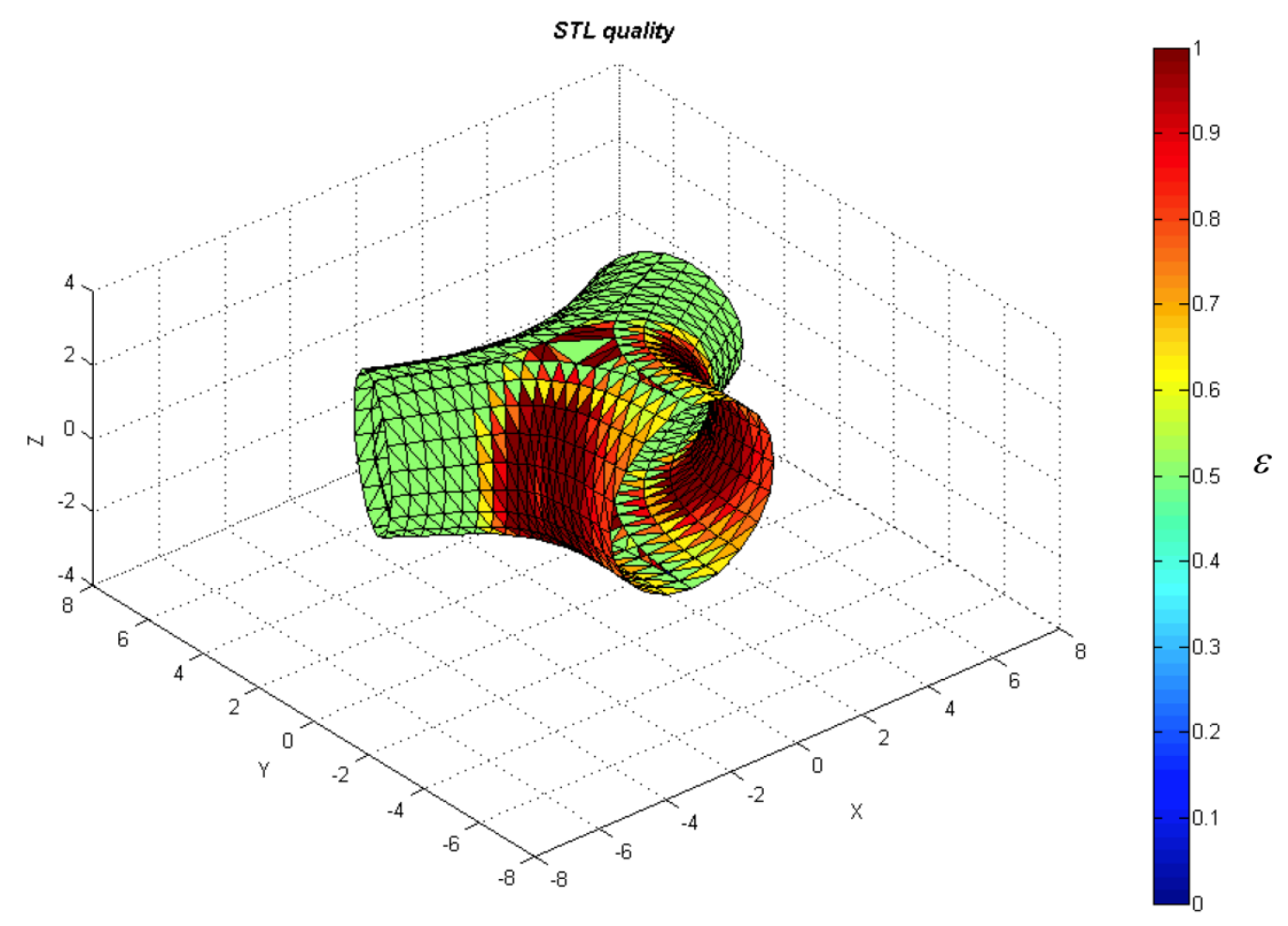

Figure 10. A STL mesh of a bifurcation junction designed using the parametric model.

\section{Design of bifurcation junction}

It is widely recognized that local geometries of a vascular bifurcation, such as bifurcation angles, junction curvatures and branching, are major features of the arterial system $[14,35]$. Previous work $[15,16,35]$ have identified that the vascular WSS has a major effect on the formation of haemodynamic diseases, such as atherosclerotic lesions and Saccular aneurysms although an exact relationship is still uncertain. The maximum curvature of the junction is the most important factor that influences WSS. High curvature also leads to stress concentration which weakens the system mechanically $[9,15,16,35]$. The volume of the junction is another important factor in haemodynamics $[11,12]$. A too large volume leads to local recirculation of the blood $[11,12]$. The basic principle for a good junction design is therefore to ensure that the volume remains in a desired narrow range while limiting 
the maximum curvature. The exact range and limit depend on specific applications. All the parameters in the model have an effect on the volume $V$ and the maximum curvature $C_{\max }$. It is convenient to normalise these parameters using the parent vessel diameter $D_{p}$ such that

$$
\bar{D}_{d 1}=\frac{D_{d 1}}{D_{p}}, \bar{D}_{d 2}=\frac{D_{d 2}}{D_{p}}, \bar{d}_{p}=\frac{d_{p}}{D_{p}}, \bar{d}_{d 1}=\frac{d_{d 1}}{D_{p}}, \bar{d}_{d 2}=\frac{d_{d 2}}{D_{p}},
$$

and that

$$
\begin{gathered}
\bar{C}_{\max }=\frac{C_{\max }}{\frac{1}{D_{p}}}, \\
\bar{V}=\frac{V}{D_{p}^{3}} .
\end{gathered}
$$

It is further defined that

$$
\begin{gathered}
\phi_{\text {total }}=\phi_{1}+\phi_{2}, \\
\alpha=\frac{\min \left(\phi_{1}, \phi_{2}\right)}{\max \left(\phi_{1}, \phi_{2}\right)}, \\
\beta=\frac{\bar{D}_{d 1}}{\bar{D}_{d 2}}
\end{gathered}
$$

and

$$
\gamma=\frac{\bar{d}_{d 1}}{\bar{d}_{d 2}}
$$

Thus, there are nine non-dimensional parameters, including $\phi_{\text {total }}, \alpha, \beta, \gamma, \bar{d}_{p}, \bar{d}_{d 1}+$ $\bar{d}_{d 2}, u_{p}, u_{d 1}$, and $u_{d 2}$, that determine $\bar{C}_{\max }$ and $\bar{V}$. In this section a systematic 
parametric study is carried out to establish a set of simple rules to achieve a balance between $V$ and $C_{\max }$. A parametric map, which can be used as a guide for designers, is provided based on the parametric study.

\subsection{Parametric analysis and design rules}

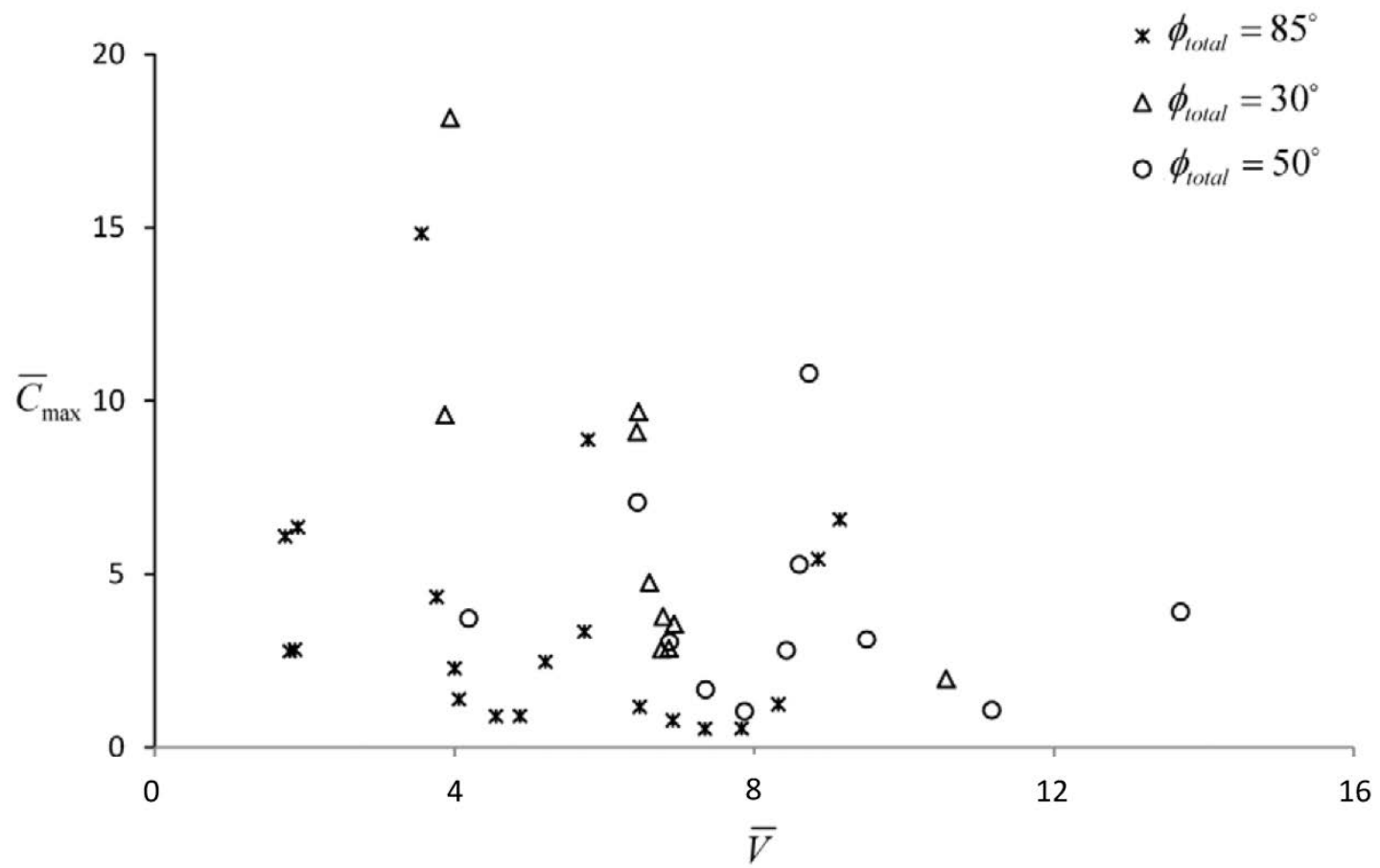

Figure 11. A $\bar{C}_{\max }-\bar{V}$ map for random parameters. .

Figure 11 shows $\bar{C}_{\max }$ and $\bar{V}$ calculated using a random set of parameters for three bifurcation angles of $\phi_{\text {total }}=30^{\circ}, \phi_{\text {total }}=50^{\circ}$, and $\phi_{\text {total }}=85^{\circ}$. The numerical results seem to suggest that there is no general correlation between $\bar{C}_{\max }$ and $\bar{V}$. However detailed analysis reveals some simple trends on how the model parameters affect the values of $\bar{C}_{\max }$ and $\bar{V}$. Because $\beta=\frac{D_{d 1}}{D_{d 2}}$, it is natural to use 


$$
\gamma=\frac{\bar{d}_{d 1}}{\bar{d}_{d 2}}=\frac{1}{\beta} .
$$

Table 1 presented cases for fixed values of $\phi_{\text {total }}=85^{\circ}$ and $\beta=1$. It can be observed from table 1 that $\bar{C}_{\max }$ is insensitive to varying the values of $\alpha, u_{p}$ and $\bar{d}_{p}$. This observation is also true for cases of other values of $\phi_{\text {total }}$ and $\beta$ which are not shown in the Table. It is recommended that fixed values are assigned to $u_{p}$ and $\bar{d}_{p}$ while $0 \leq \alpha \leq 1$ is used for different applications. This leads to the first simple rule of design:

- $\quad$ one can use values of $0 \leq \alpha \leq 1, u_{p}=1, \bar{d}_{p}=2$ and $\gamma=\frac{1}{\beta}$ for all applications.

Table 1. Values of $\bar{C}_{\max }$ and $\bar{V}$ calculated using different values of the model parameters, $\phi_{\text {total }}=85^{\circ}, \beta=1$ and $\gamma=1$

\begin{tabular}{|c|c|c|c|c|c|c|c|c|}
\hline $\begin{array}{l}\text { Case } \\
\text { NO. }\end{array}$ & $\alpha$ & $\bar{d}_{p}$ & $\bar{d}_{d 1}+\bar{d}_{d 2}$ & $u_{p}$ & $u_{d 1}$ & $u_{d 2}$ & $\bar{C}_{\max }$ & $\bar{V}$ \\
\hline 1 & \multirow{11}{*}{1} & \multirow{11}{*}{3} & \multirow{11}{*}{6} & \multirow{11}{*}{1} & \multicolumn{2}{|c|}{0.25} & 6.570 & 9.1 \\
\hline 2 & & & & & \multicolumn{2}{|c|}{0.3} & 5.430 & 8.9 \\
\hline 3 & & & & & \multicolumn{2}{|c|}{0.4} & 1.238 & 8.3 \\
\hline 4 & & & & & \multicolumn{2}{|c|}{0.5} & 0.537 & 7.8 \\
\hline 5 & & & & & \multicolumn{2}{|c|}{0.6} & 0.532 & 7.3 \\
\hline 6 & & & & & \multicolumn{2}{|c|}{0.7} & 0.778 & 6.9 \\
\hline 7 & & & & & \multicolumn{2}{|c|}{0.8} & 1.163 & 6.5 \\
\hline 8 & & & & & \multicolumn{2}{|c|}{1} & 3.338 & 5.7 \\
\hline 9 & & & & & 0.8 & 0.5 & 0.525 & 8.5 \\
\hline 10 & & & & & 0.85 & 0.535 & 0.778 & 6.9 \\
\hline 11 & & & & & 1 & 0.59 & 1.163 & 6.5 \\
\hline 12 & $\frac{1}{16}$ & \multirow{2}{*}{3} & \multirow{2}{*}{6} & \multirow{2}{*}{1} & \multirow{2}{*}{\multicolumn{2}{|c|}{0.6}} & 0.532 & 7.0 \\
\hline 13 & $\frac{3}{14}$ & & & & & & 0.532 & 7.15 \\
\hline
\end{tabular}




\begin{tabular}{|c|c|c|c|c|c|c|c|}
\hline 14 & $\frac{6}{11}$ & & & & & 0.532 & 7.32 \\
\hline 15 & \multirow{12}{*}{1} & 1 & \multirow{6}{*}{6} & \multirow{3}{*}{1} & \multirow{6}{*}{0.6} & 0.532 & 5.5 \\
\hline 16 & & 1.5 & & & & 0.532 & 6.15 \\
\hline 17 & & 2 & & & & 0.532 & 6.33 \\
\hline 18 & & 2 & & 0.7 & & 0.532 & 6.69 \\
\hline 19 & & 2 & & 0.5 & & 0.532 & 6.94 \\
\hline 20 & & 2 & & 0.3 & & 0.532 & 7.19 \\
\hline 21 & & 2 & 10 & 1 & 0.6 & 0.295 & 11.4 \\
\hline 22 & & 2 & 8 & 1 & 0.6 & 0.379 & 8.70 \\
\hline 23 & & 2 & 4 & 1 & 0.6 & 0.893 & 4.29 \\
\hline 24 & & 2 & 3.5 & 1 & 0.6 & 1.075 & 3.84 \\
\hline 25 & & 2 & 3 & 1 & 0.6 & 1.35 & 3.40 \\
\hline 26 & & 2 & 2 & 1 & 0.6 & 2.765 & 2.6 \\
\hline
\end{tabular}

Next we focus on cases $1-8$ for which only $u_{d 1}$ (set as equal to $u_{d 2}$ ) is varied. Their corresponding $\bar{C}_{\max }$ and $\bar{V}$ are plotted in figure 12.

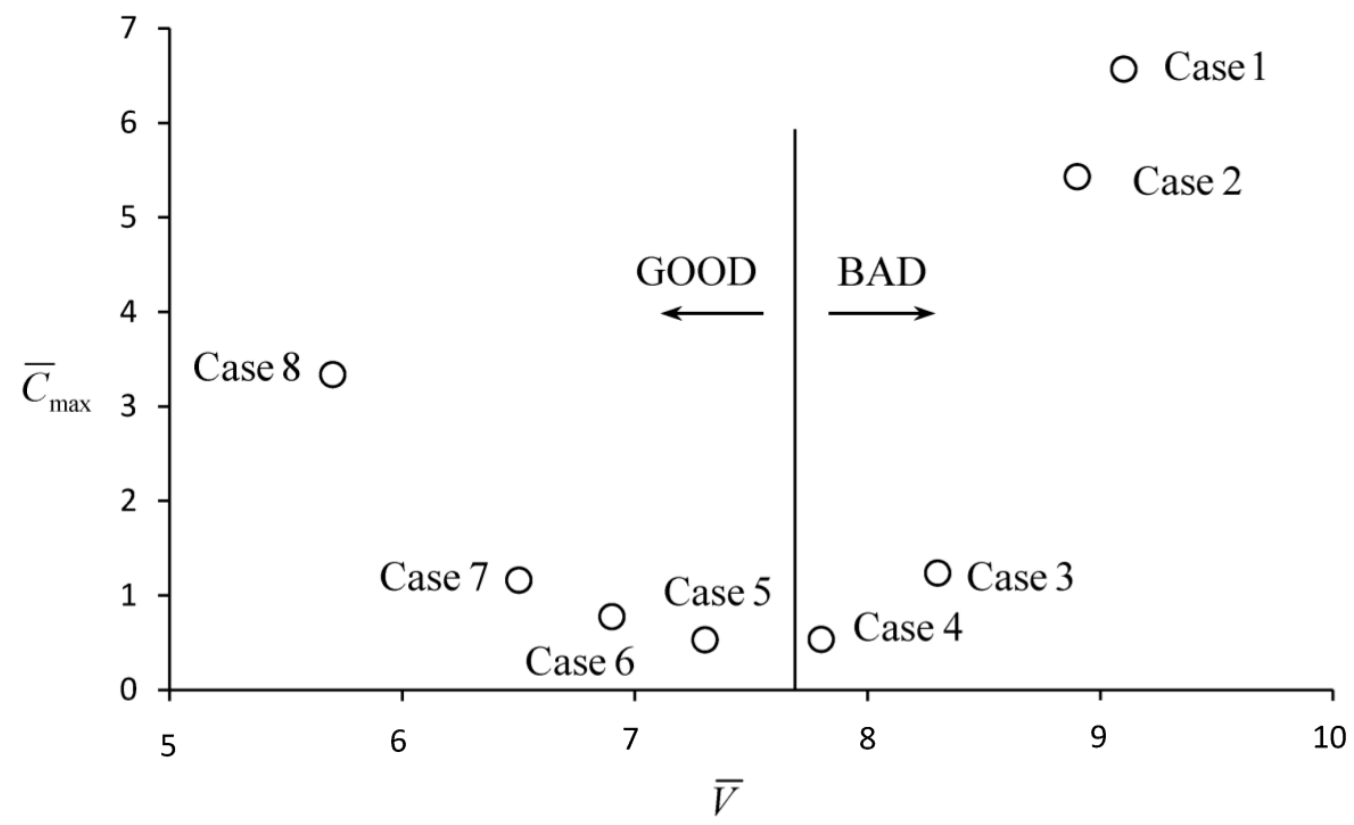

Figure 12. A $\bar{C}_{\max }-\bar{V}$ plots for cases 1 to 8 . 
It can be observed that as $u_{d 1}$ is reduced, $\bar{C}_{\max }$ firstly reduces as $\bar{V}$ increases from cases 8 to 5 and then increases from cases 4 to 1 . The increase of $\bar{C}_{\max }$ corresponding to an increasing $\bar{V}$ is against common sense.

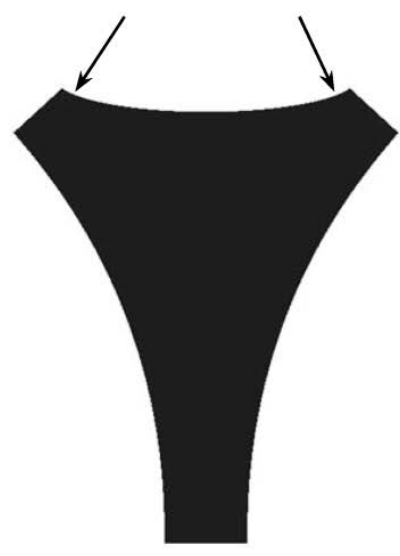

Case 1

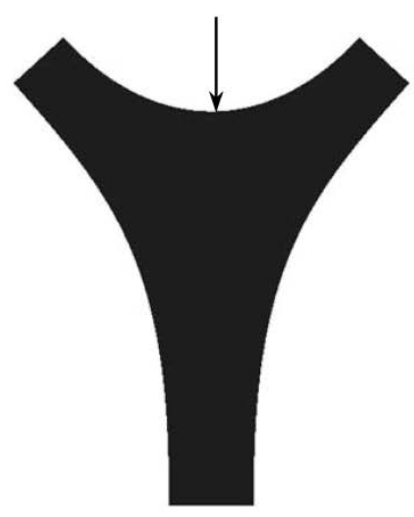

Case 5

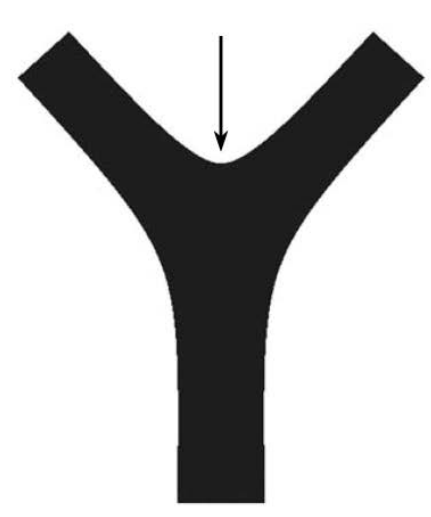

Case 8

Figure 13. Bifurcation junctions designed by the parametric model using different parameters: case 1 , case 5 and case 8 .

Figure 13 shows the actual junctions of cases 1, 5 and 8 . The locations of maximum curvature are pointed out using the arrows. The figure shows that the maximum curvature for case 1 occurs at the connecting parts between the junction and the rest of the vessel. The same is true for cases 2,3 and 4 . This is because when very small values of $u_{d 1}$ and $u_{d 2}$ are used, the two control points on the Bezier curve $l_{2}$ are very close to the two end points giving limited lengths for $l_{2}$ to change its tangent value from one end to another. These are obviously bad designs and should be ruled out as indicated in figure 12. This leads to the second simple rule of design:

- one should always avoid using small values for both $u_{d 1}$ and $u_{d 2}$, less than 0.6 for $\phi_{\text {total }}=85^{\circ}$, for example. 
Figure 14 plots $\bar{C}_{\max }$ versus $\bar{V}$ for cases $17,21,22,23,24,25$, and 26 in Table 1. In these cases all the parameters are fixed except for $\bar{d}_{d 1}+\bar{d}_{d 2}$.

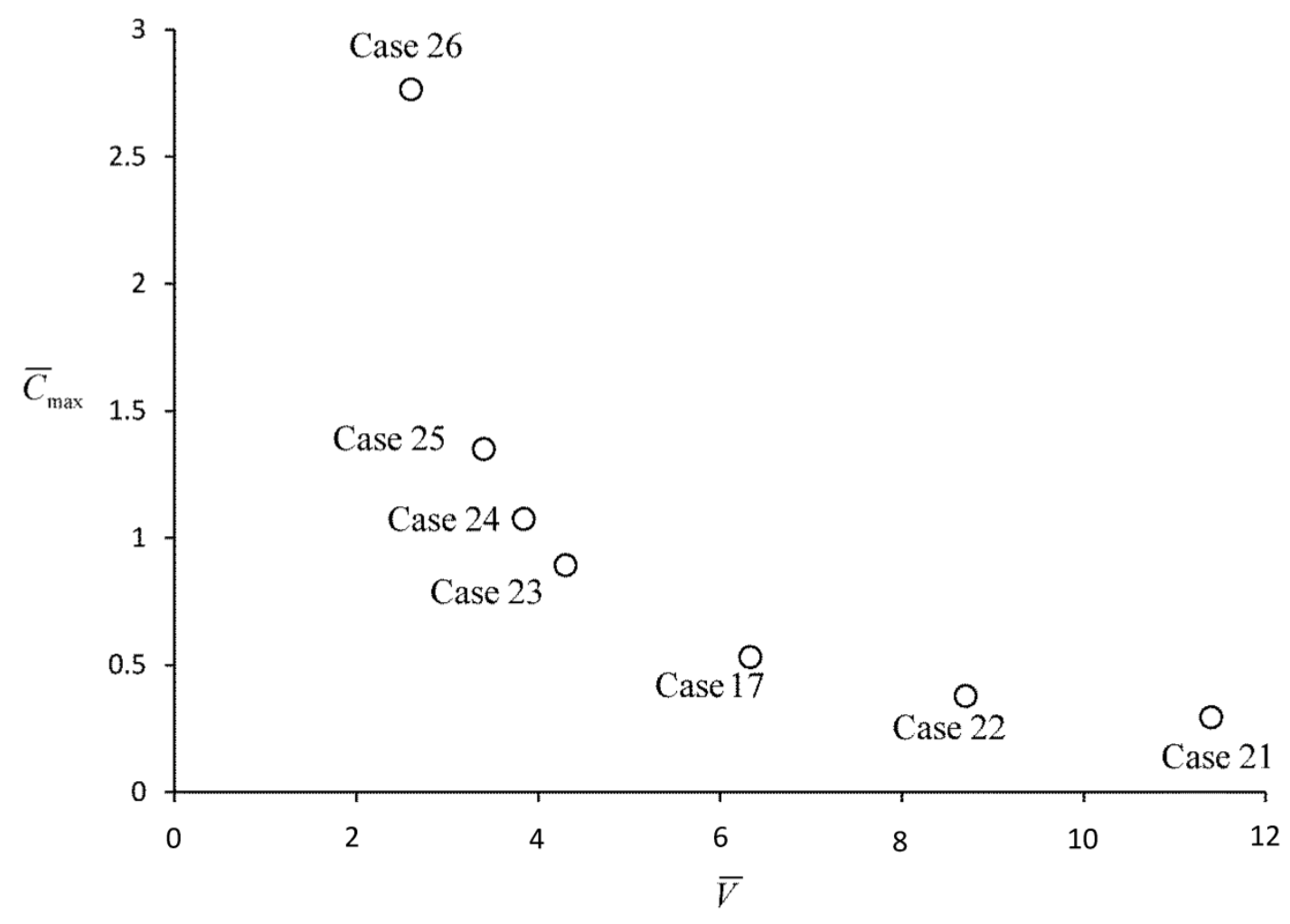

Figure 14. $\bar{C}_{\max }-\bar{V}$ plots for cases $17,21,22,23,24,25$, and 26.

The values of $\bar{d}_{d 1}+\bar{d}_{d 2}$ increases from 2 for case 26 to 10 for case 21 . It can be observed that both $\bar{C}_{\max }$ versus $\bar{V}$ are sensitive to the values of $\bar{d}_{d 1}+\bar{d}_{d 2}$. Not shown in the figure, $\bar{V}$ is nearly linearly proportional to $\bar{d}_{d 1}+\bar{d}_{d 2}$ with $R^{2}=0.9936$. Figure 14 also suggests that $\bar{d}_{d 1}+\bar{d}_{d 2}$ should be less than 6 (corresponding case 17 ) beyond which a larger volume does not lead to significant reduction in $\bar{C}_{\max }$. On the other hand, $\bar{d}_{d 1}+\bar{d}_{d 2}$ should also be larger than 4 , to avoid the large $\bar{C}_{\max }$ as shown on the left side of the figure. This is because the Bezier curve $l_{2}$ has insufficient length to change its curvature from one end to another moderately if $\bar{d}_{d 1}+\bar{d}_{d 2}$ is less 
than 4. This is also true for other values of $\phi_{\text {total }}$ and $\beta$. It is therefore recommended to use $\bar{d}_{d 1}+\bar{d}_{d 2}=6$. We therefore have the third simple design rule

- one should use $\bar{d}_{d 1}+\bar{d}_{d 2}=6$ for all applications.

Using the above three simple rules, it is then convenient to manipulate the values of $\bar{u}_{d 1}$ and $\bar{u}_{d 2}$ in the junction design in order to achieve a targeted set of values of $\bar{C}_{\max }$ and $\bar{V}$. Our numerical study shows that $\bar{V}$ is not over-sensitive to the change in $\bar{u}_{d 1}$ and $\bar{u}_{d 2}$ while $\bar{C}_{\max }$ is rather sensitive to the change in $\bar{u}_{d 1}$ and $\bar{u}_{d 2} \cdot \bar{u}_{d 1}$ and $\bar{u}_{d 2}$ are therefore good design variables because they allow one to select desired $\bar{C}_{\max }$ within keeping $\bar{V}$ within a narrow range. This leads to the fourth simple design rule

- one should use $\bar{u}_{d 1}$ and $\bar{u}_{d 2}$ as the fundamental design variable in order to achieve a targeted maximum curvature while keeping the junction volume within a narrow range.

\subsection{The parametric map and an application}

If we exclude the cases for which the maximum curvature occurs at the connecting part between the junction and rest of the vessel from Table 1 , then the $\bar{C}_{\max }$ versus $\bar{V}$ plot follows a clear trend that an increasing volume leads to a decreasing curvature as shown in figure 14. This is in strong contrast to Figure 11. 


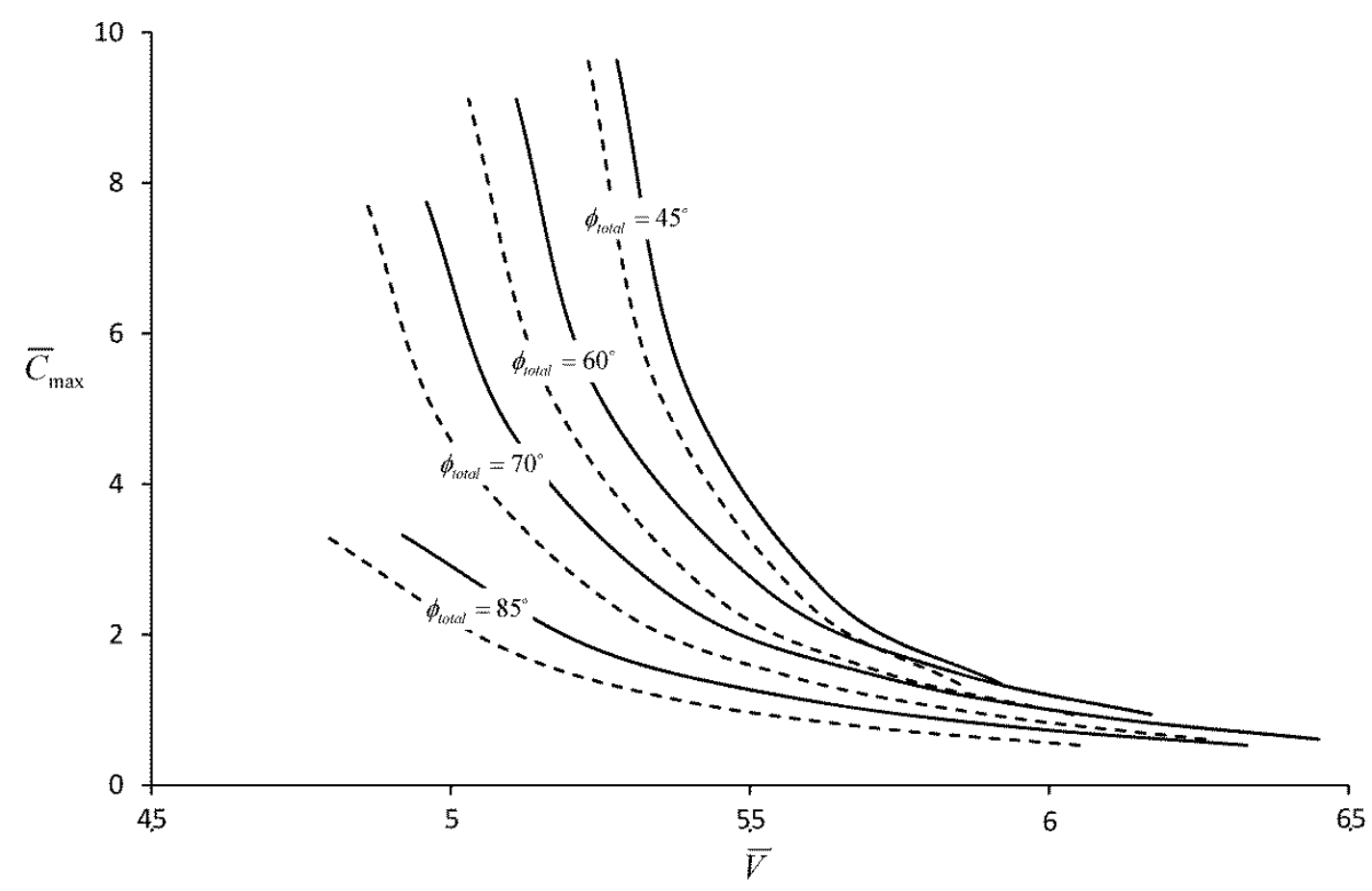

Figure 15. $\bar{C}_{\max }-\bar{V}$ plots for different bifurcation angles: $\phi_{\text {total }}=45^{\circ}, \phi_{\text {total }}=60^{\circ}$,

$$
\phi_{\text {total }}=70^{\circ} \text { and } \phi_{\text {total }}=85^{\circ} \text {. }
$$

Figure 15 shows the calculated $\bar{C}_{\max }$ versus $\bar{V}$ using these exclusions for four different values of $\phi_{\text {total }}$ and a fixing value of $\beta=1$. Unlike in figure $14, \bar{d}_{d 1}+\bar{d}_{d 2}$ is set as 6 and all the simple design rules are implemented in figure 15. $\bar{u}_{d 1}$ and $\bar{u}_{d 2}$ are used as the fundamental design variables while $\alpha$ is also varied as the secondary design variable. For each bifurcation angle, $\phi_{\text {total }}$, one dashed line and one solid line are plotted reflecting a band corresponding to different values of $\alpha$. The band is rather narrow showing the insensitivity to $\alpha$. Figure 15 can be used as a design guide to find the possible combinations of $\bar{C}_{\max }$ and $\bar{V}$. Figure 16 shows the same plot but for a full range of values of $\phi_{\text {total }}$. 


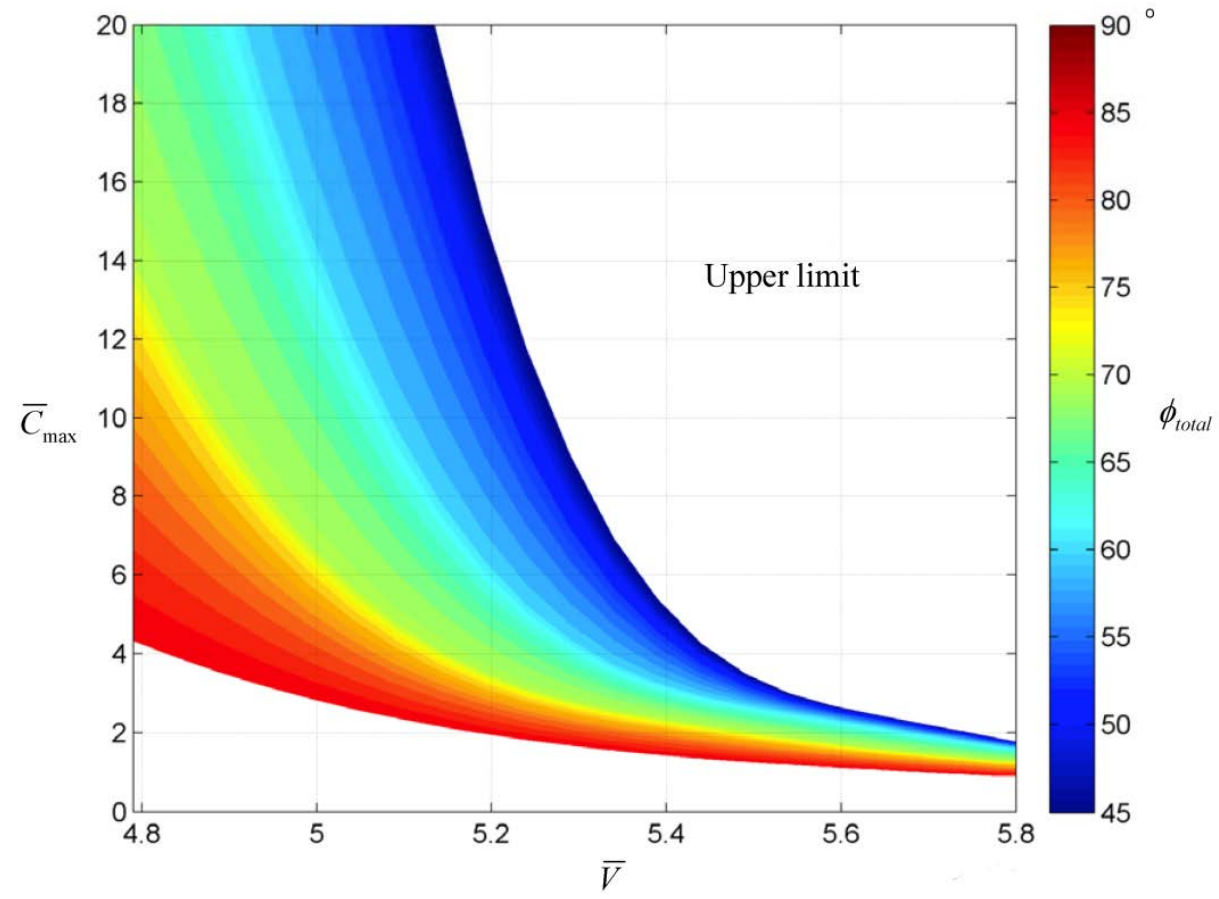

(a)

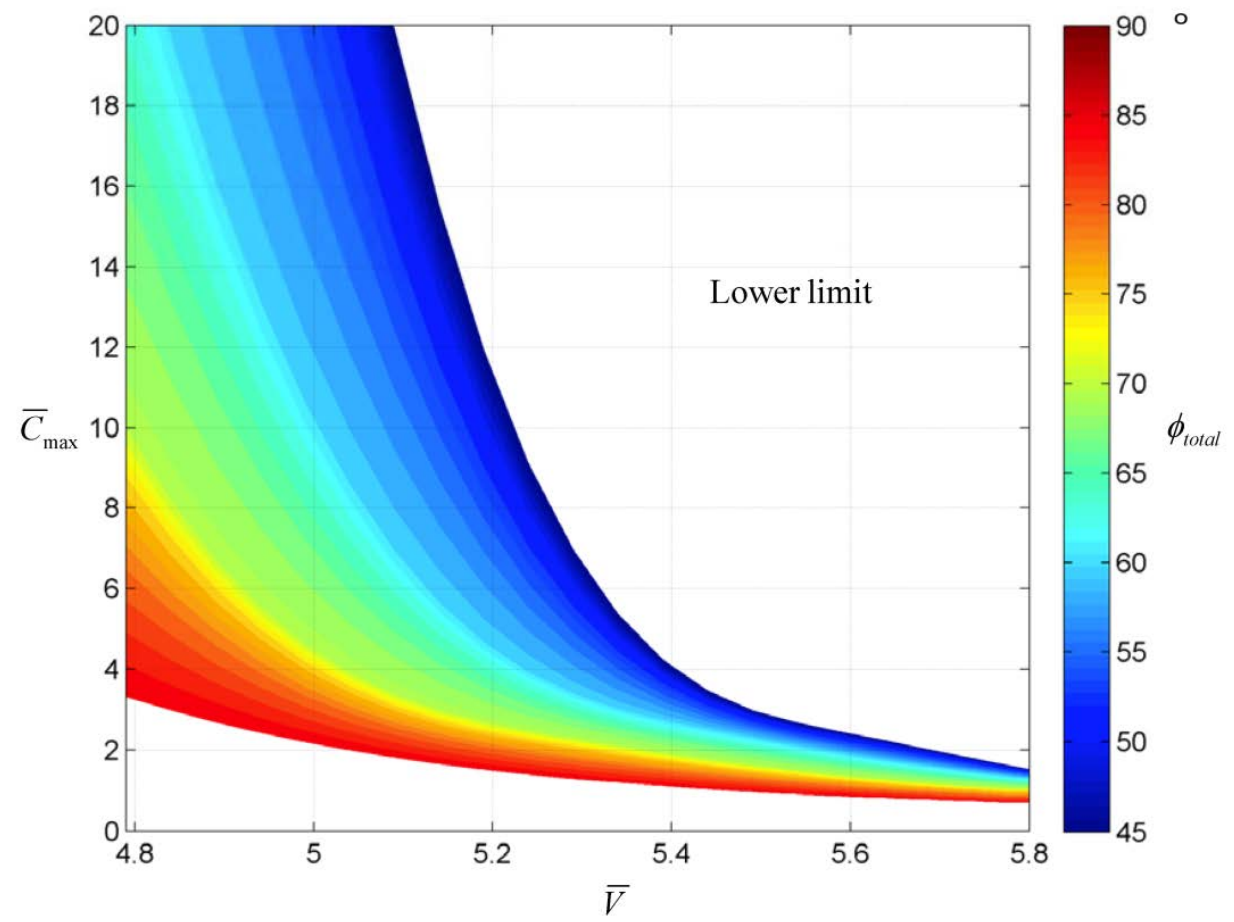

(b) 
Figure 16. A generalised parametric map: (a) the upper limit, and (b) the lower limit. Figure 16 (a) show the upper limit (corresponding to the solid line in Fig.15) while Figure 16 (b) shows the lower limit (corresponding to the dash line in Fig. 15). The colour bars indicate the total bifurcation angles $\phi_{\text {total }}$.

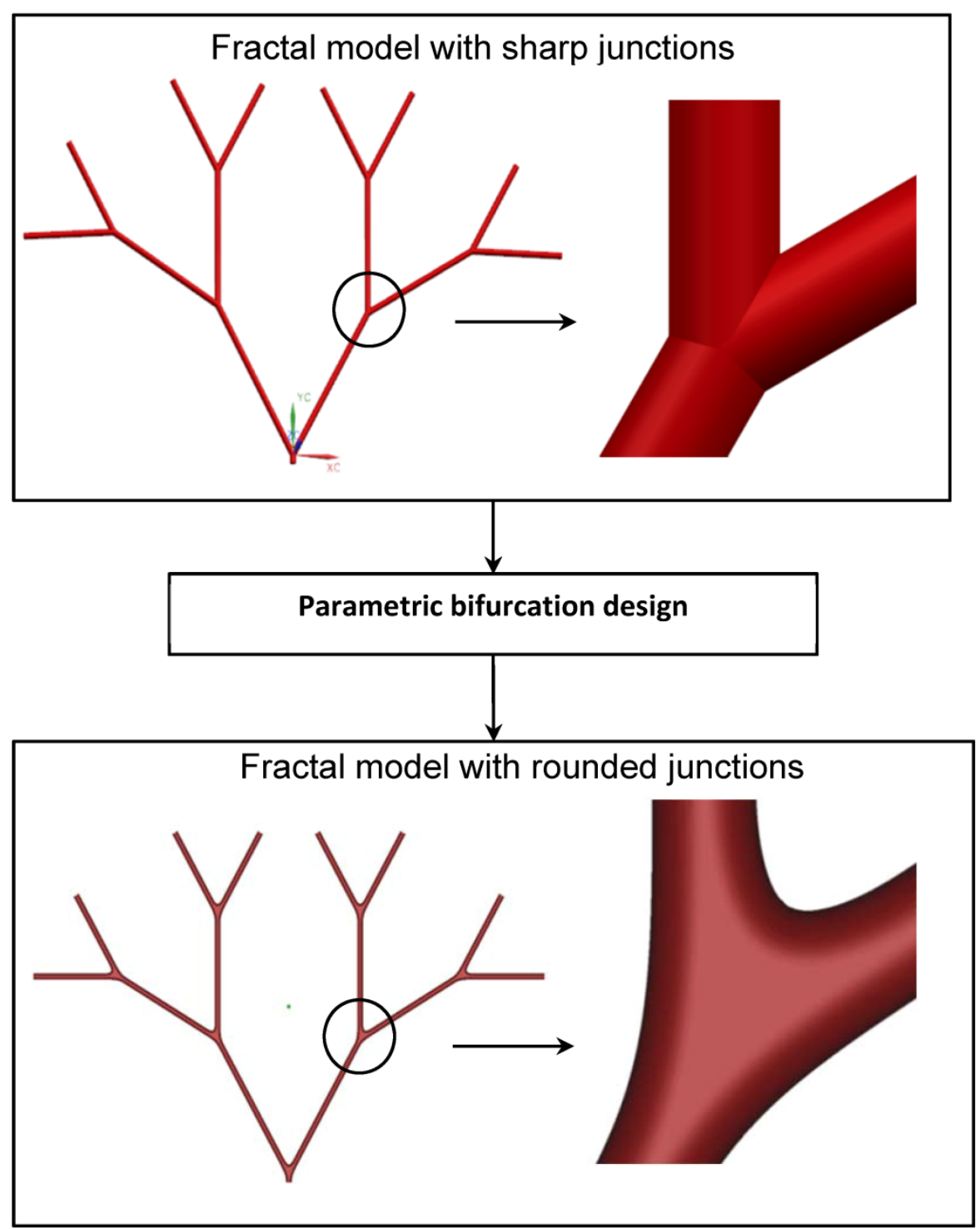

Figure 17. A fractal vascular model using the parametric bifurcation model. 


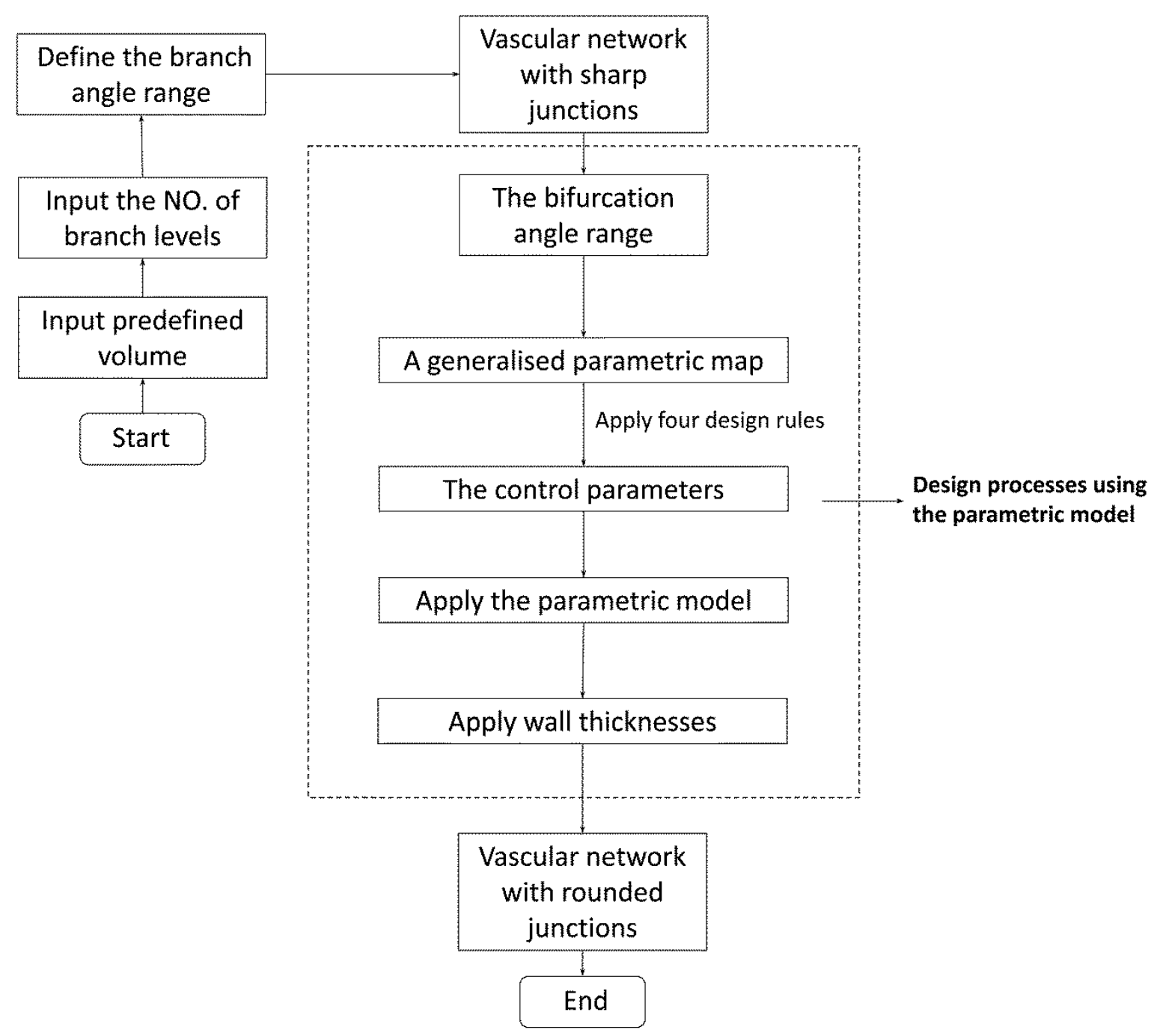

Figure 18. The flow chart for a fractal vascular tree design.

An application of using the parametric junction model is shown in figure 17 according to the flow chart shown in figure 18 . Using the parametric map of figure 16 , one can easily find a desired pair of $\bar{C}_{\max }$ and $\bar{V}$ to design a bifurcation junction. Its corresponding model parameters can be obtained according to the simple design rules. An error free STL file is generated from the parametric model for this application which is checked and verified by Magics ${ }^{\circledR}$. The result shows that approximately 7000 extra triangles in an output STL file of rounded network are created while the original vascular network yields around 30000 triangles, using a very fine mesh. 


\section{Concluding remarks}

AM techniques play an important part in the field of tissue engineering. It enables the manufacture of complex three dimensional scaffolds or bifurcation vascular networks with controllable geometries. In this paper, we introduce simplifications and integrations of existing mathematical algorithms in the literature that can be conveniently used for modelling bifurcation junctions in tissue engineering. The simplicity of the model enables a focus on key experimental variables, the maximum curvature and the volume of the bifurcation, required by researchers e.g. for the ArtiVasc 3D project. In the parametric analysis, nine parameters were identified and non-dimensionlised which would affect both the maximum curvature and the volume of the junction. Four simple design rules were obtained from these studies, following which, a maximum curvature - volume map was presented. Applying this map, a suitable curvature at the apex and its corresponding volume can be achieved. The work presented in this paper therefore outlined a simple algorithm for modelling the bifurcation junctions and provided a simple set of rules and guide on how to choose the parameters in the model.

\section{Acknowledgements}

This work is part of the project ArtiVasc 3D (http://www.artivasc.eu/). It is financially supported by the European Union's Seventh Framework Programme (FP/2007-2013) under grant agreement No. 263416 (ArtiVasc 3D).

\section{References}

[1] R.A. Kamel, J.F. Ong, E.Eriksson, J.P.Junker, E.J.Caterson, Tissue Engineering of Skin, J. Am. Coll. Surgeons. 217( 2013) 533-55. 
[2] http://www.nc3rs.org.uk.

[3] F.R.A.J. Rose, R.O.C.Oreffo, Bone Tissue Engineering: Hope vs Hype, Biochem. Bioph. Res. Co. 292 (2002) 1-7.

[4] J.Patrick, W.Charles, Adipose tissue engineering: The future of breast and soft tissue reconstruction following tumor resection, Semin. Surg. Oncol.19 (2000) 30211.

[5] R.Landers, A.Pfister, U.Hubner, H.John, R.Schmelzeisen, R. Hulhaupt, Fabrication of soft tissue engineering scaffolds by means of rapid prototyping techniques, J. Mater. Sci. 37 (2002) 3107-16.

[6] ArtiVasc 3D - Development of artificial vaskularised skin.

[7] G.S. Kassab, C.A. Rider, N.J. Tang, Y-CB. Fung, Morphometry of pig coronary arterial trees, Am J Physiol. 265 (1993) H350-65.

[8] J. Ravensbergen, J.K.B. Krijger, B.Hillen, H. Hoogstraten, Merging flows in an arterial confluence: the vertebra-basilar junction, J. Fluid Mech, 304 (1995) 119-41.

[9] J. Ravensbergen, J.K.B. Krijger, A.L. Verdaasdonk, B. Hillen, H.W. Hoogstraten, The influence of the blunting of the apex on the flow in a Vertebro-Basilar junction model, J. Biomech. Eng. 119 (1997) 195-205.

[10] I. Gibson, D.W. Rosen, B. Stucker, Additive manufacturing technologies: Rapid prototyping to direct digital manufacturing, Springer, 2010.

[11] Q. Long, X.Y. Xu, M. Bourne, T.M. Griffith, Numerical study of blood flow in an anatomically realistic Aorto-Iliac bifurcation generated from MRI data, Magnet. Reson. Med, 43 (2000) 565-76.

[12] H. Meng, Z. Wang, Y. Hoi, L. Gao, E. Metaxa, D. Swartz, et al., Complex hemodynamics at the apex of an arterial bifurcation induces vascular remodelling resembling cerebral aneurysm initiation, Stroke 38 (2007) 1924-31. 
[13] G. Foutrakis, Yonas H, Sclabassi R. Saccular Aneurysm formation in curved and bifurcating arteries. American Journal of Neuroradiology; 1999. p. 1309-17.

[14] I. Marshall, S. Zhao, P. Papathanasopoulou, P. Hoskins, X.Y. Xu, MRI and CFD studies of pulsatile flow in healthy and stenosed carotid bifurcation models, $\mathrm{J}$. Biomech; 37 (2004) 679-87.

[15] U. Kohler, I. Marshall, M.B. Robertson, Q. Long, X.Y. Xu, P.R. Hoskins, MRI measurement of wall shear stress vectors in bifurcation models and comparison with CFD predictions, Jmri-J. Magn. Reson. Im, 14 (2001) 563-73.

[16] G, Coppola, C.G. Caro, Arterial geometry, flow pattern, wall shear and mass transport: potential physiological significance, J. R. Soc. Interface, (2008) 1-10.

[17] J. Bloomenthal, Skeletal Design of Natural Forms. Thesis; Department of Computer Science, University of Calgary, 1995.

[18] J.Kretschmer, C.Godenschwager, B.Preim, M.Stamminger, Interactive patientspecific vascular modelling with sweep surfaces. IEEE. T. Vis. Comput. G. 19 (2013) 2828-37.

[19] Y. Zhang, Y. Bazilevs, S. Goswami, C.L. Bajaj, Patient - specific vascular NURBS modelling for isogeometric analysis of blood flow, Comput. Method. Appl. M. 196 (2007) 2943-59.

[20] O. Gourmel, L. Barthe, M-P. Cani, B. Wyvill, A. Bernhardt, M. Paulin, et al., A gradient-based implicit blend, ACM. T. Graphic, 32 (2013) 12:1-12:12.

[21] R. Krasauskas. Branching blend of natural quadrics based on surfaces with rational offsets. Comput. Aided. Geom. D., 25 (2008) 332-41.

[22] Y. Cai, X. Ye, C. Chui, J.H. Anderson, Constructive algorithms of vascular network modeling for training of minimally invasive catheterization procedure, Adv. Eng. Softw. 34 (2003) 439-50. 
[23] X. Ye, Y. Cai, C. Chui, J.H. Anderson, Constructive modeling of G1 bifurcation, Comput. Aided. Geom. D. 19 (2002) 513-31.

[24] E. Ferley, M.P. Cani-Gascuel, D. Attali, Skeletal reconstruction of branching shapes, Comput. Graph. Forum. 16 (1997) 283-93.

[25] J. Bloomenthal, K. Shoemake. Convolution surfaces, ACM SIGGRAPH Computer Graphics, 25 (1991) 251-6.

[26] J. Bloomenthal, Bulge elimination in implicit surface blends, Implicit Surfaces, 1995 7-20.

[27] C. Galbraith, P. Macmurchy, B. Wyvill. Blobtree trees, Proceedings of Computer Graphics International, (2004) 78-85.

[28] H. Zakaria, A.M. Robertson, C.W. Kerber, A parametric model for studies of flow in arterial bifurcations, Ann. Biomed. Eng. 36(2008) 1515-30.

[29] Bastl B, Juttler B, Lavicka M, Schulz T. Blends of canal surfaces from polyhedral medial transform representations. Computer Aided Design. 43 (2011) 1477-84.

[30] E.E. Hartquist, J.P. Menon, K. Suresh, H.B. Voelcker, J. Zagajac, A computing strategy for applications involving offsets, sweeps, and Minkowski operations. Comput Aided Design. 31 (1999) 175-83.

[31] I.D. Faux, M.J. Pratt, Computational geometry for design and manufacture, Halsted Press, New York, 1979.

[32] C.D. Murray, The physiological principle of minimum work. I. the vascular system and the cost of the blood volume, P. Natl. Acad. Sci. Usa. 12 (1926) 299-304. [33] M. Bern, D. Eppstein, Mesh generation and optimal triangulation in: D.Z Du, F. Hwang, Computing in Euclidean geometry, World Scientific, 1992 23-90.

[34] G. Barequet, M. Sharir, Filling gaps in the boundary of a polyhedron, Comput. Aided. Geom. D, 13 (1995) 207-29. 
[35] C.G. Caro, Discovery of the role of wall shear in atherosclerosis, Arterioscler. Thromb. Vasc. Biol, 29 (2008) 158-61.

\section{Figure captions}

Figure 1. Schematic diagram of a swept surface with a moving object $\mathbf{S}$ and a motion trajectory $\mathbf{M} ; t$ is the sweeping time.

Figure 2. Variable definitions of a) cubic Bezier curve; and b) a cloud point of a swept surface.

Figure 3. A swept surface by sweeping a changing circular cross sectional contour along a cubic Bezier developed in figure 2(b) using a linear interpolation method provided in equation (2).

Figure 4. A schematic diagram for a bifurcation junction and variable definitions.

Figure 5. A bifurcation triangle $P_{p} P_{d 1} P_{d 2}$, corresponding unit vectors: $v_{p}, v_{d 1}$ and $v_{d 2}$, three control points: $Q_{p}, Q_{d 1}$ and $Q_{d 2}$, and bifurcation angles: $\phi_{1}$ and $\phi_{2}$.

Figure 6. Curved bifurcation triangles $\left(l_{1}, l_{2}, l_{3}\right)$ representing by cubic Bezier curves with different sets of control parameters: from outer triangle to inner triangles, values of control parameters, in turn, are $u_{p}=u_{d 1}=u_{d 2}=0, u_{p}=u_{d 1}=u_{d 2}=0.3$, $u_{p}=u_{d 1}=u_{d 2}=0.7$, and $u_{p}=u_{d 1}=u_{d 2}=1.0$

Figure 7. The swept semi-tubular bifurcation junction.

Figure 8. A polygon hole filling of a planar hole $\omega=0.01, \varepsilon=0.5494$.

Figure 9. A polygon hole filling of a non-planar hole $\omega=0.01, \varepsilon=0.5789$.

Figure 10. A STL mesh of a bifurcation junction designed using the parametric model.

Figure 11. A $\bar{C}_{\max }-\bar{V}$ map for random parameters. . 
Figure 12. A $\bar{C}_{\max }-\bar{V}$ plots for cases 1 to 8 .

Figure 13. Bifurcation junctions designed by the parametric model using different parameters: case 1 , case 5 and case 8 .

Figure 14. $\bar{C}_{\max }-\bar{V}$ plots for cases $17,21,22,23,24,25$, and 26.

Figure 15. $\bar{C}_{\max }-\bar{V}$ plots for different bifurcation angles: $\phi_{\text {total }}=45^{\circ}, \phi_{\text {total }}=60^{\circ}$, $\phi_{\text {total }}=70^{\circ}$ and $\phi_{\text {total }}=85^{\circ}$.

Figure 16. A generalised parametric map: (a) the upper limit, and (b) the lower limit. Figure 17. A fractal vascular model using the parametric bifurcation model.

Figure 18. The flow chart for a fractal vascular tree design. 